\title{
FLUCTUATING SYSTEMS UNDER CYCLIC PERTURBATIONS: RELATION BETWEEN ENERGY DISSIPATION AND INTRINSIC RELAXATION RATES
}

\author{
Fabrizio Camerin \\ Department of Chemical Sciences, University of Padova, via Marzolo 1, I-35131, \\ Padova, Italy. Presently a scholar at the University of Rome "La Sapienza" \\ partecipating in the Erasmus Mundus Joint Master Degree program AtoSiM \\ Diego Frezzato* \\ Department of Chemical Sciences, University of Padova, via Marzolo 1, \\ I-35131, Padova, Italy. ${ }^{*}$ Corresponding author. Email: diego.frezzato@unipd.it
}

(Dated: July 22, 2016)

\begin{abstract}
This study focuses on fluctuating classical systems in contact with a thermal bath, and whose configurational energetics undergoes cyclic transformations due to interaction with external perturbing agents. Under the assumptions that the configurational dynamics is a stochastic Markov process in the overdamped regime and that the non-equilibrium configurational distribution remains close to the underlying equilibrium one, we derived an analytic approximation of the average dissipated energy per cycle in the asymptotic limit (i.e., after many cycles of perturbation). The energy dissipation is then readily translated into average entropy production, per cycle, in the environment. The accuracy of the approximation was tested by comparing the outcomes with the exact values obtained by stochastic simulations of a model case: a "particle on a ring" which fluctuates in a bi-stable potential perturbed in two different ways. As pointed out in previous studies on the stochastic resonance phenomenon, the dependence of the average dissipation on the perturbation period may unveil the inner spectrum of system's fluctuation rates. In this respect, the analytical approximation presented here makes it possible to unveil the connection between average dissipation, intrinsic rates/modes of fluctuation of the system at the unperturbed equilibrium, and features of the perturbation itself (namely, the period of the cycle and the projections of the energy perturbation over the system's modes). The possibilities of employing the analytical results as a guide to devising and rationalizing a sort of "spectroscopic calorimetry" experiment, and of employing them in strategies aiming to optimize the system's features on the basis of a target average dissipation, are briefly discussed.
\end{abstract}




\section{INTRODUCTION AND OUTLINE OF THE WORK}

This work deals with classical systems subjected to an externally driven periodic perturbation of the configurational energetics while they fluctuate in contact with a thermal bath. Namely, it focuses on the amount of energy which is dissipated per cycle of the perturbation, and, in particular, on its limit value asymptotically attained after many cycles. Our main objective was to elaborate an approximate expression which relates the average dissipation to the intrinsic relaxation modes/rates of the unperturbed system at equilibrium and to the features of the energy perturbation (i.e., functional form and period). That approximation, Eq. 21 in the following, is accurate when the non-equilibrium configurational distribution of the system differs slightly from the "underlying" one corresponding to a virtual equilibration with the external perturbation.

Based on this premise, our study fits into so-called "stochastic thermodynamics" [1], one of the most exciting branches of physical-chemical sciences being studied over the last two decades. In this context, the quantities of work and heat exchanged between the system and the environment become stochastic variables since the actual trajectory followed by the uncontrolled degrees of freedom of the system is, in itself, stochastic. Insights on the statistical properties of these quantities of energy have led to a number of remarkable "fluctuation theorems"; we address interested readers to reviews [2-5] and to references therein. More specifically, energy dissipation in periodically driven systems has been explored in a series of previous studies both experimentally and computationally (see, for example, refs. $[6,7])$. We would also like to mention the theoretical work of Harada and Sasa [8] who derived an equality, for Langevin systems, that connects the rate of energy dissipation at steady-state with the extent of violation of the "fluctuation-response relation"; this equality has been proven to hold even for periodically driven systems. [9] Morever, we mention the close connection, as will be clarified later, between the present study and recent developments in the build-up of the (linear) non-equilibrium thermodynamics of periodically driven systems.[10, 11]. However, to the best of our knowledge, a detailed link between the average dissipation per cycle and the internal modes/rates of fluctuation is attempted here for the first time.

Before going into the technicalities of the specific problem treated here, we will outline its essential traits and provide some introductory concepts. Concerning the units of energy adopted throughout, all quantities having physical dimension of energy (i.e., work, potential energies, free energies and derived quantities) will be implicitly given in $k_{B} T$ units, where $T$ is the absolute temperature of the thermal bath and $k_{B}$ the Boltzmann constant. All these quantities will thus be dimensionless and the reader should retrieve the physical units, if needed, by proper re-scaling. 
In all generality, let $\mathbf{x}$ be the minimal set of coordinates of configurational type which are essential to specify the actual microstate of a given system. The requirement is that the dynamics of $\mathbf{x}$, both with and without the external perturbation, is a multidimensional diffusive Markov process. [12] Accordingly, the dynamics can be modeled by means of a Langevin-like equation in the overdamped regime of motion to generate trajectories or by means of the corresponding Fokker-Planck equation in the Smoluchowski form to describe the evolution of the non-equilibrium probability distribution $p(\mathbf{x}, t)$ from a given initial profile $p(\mathbf{x}, 0)$.[12] Now consider an external agent (see remarks below) which interacts with the system so that the energy of each configuration, $V(\mathbf{x}, t),[13]$ changes deterministically in time. While the energy modulation is active, the variables $\mathbf{x}$ evolve in uncontrolled way so that a stochastic trajectory $\mathbf{x}(t)_{t r}$ develops (in what follows, the subscript " $t r$ " will label quantities referring to a single trajectory). The infinitesimal amount of thermodynamic work performed along the actual system's trajectory is identified [1, 14, 15] with the variation of the microstate energy due to the controlled external intervention, that is

$$
\delta w_{t r}(t, t+\delta t):=\delta t \times\left.\frac{\partial V(\mathbf{x}, t)}{\partial t}\right|_{\mathbf{x}=\mathbf{x}(t)_{t r}}
$$

The net work done along the trajectory, $w_{t r}$, is then obtained by summing all the infinitesimal contributions. The stochasticity of the trajectory implies that $w_{t r}$ is also a stochastic variable.

As in the usual macroscopic context,[16] an applied transformation (possibly) causes a change of the system's thermodynamic state. While the system's configuration is specified by $\mathbf{x}$, the thermodynamic state refers to the statistical distribution of the configurations at equilibrium. The well-known state-functions of macroscopic thermodynamics are then defined in terms of specific ensemble averages. For fluctuating systems in contact with a thermal bath, the key state-function is the Helmholtz free energy $A$. Other state-functions, such as the entropy $S$ and the internal energy $U$, can be obtained from the temperature dependence of $A$ by applying the proper differential interrelations. Let the system be initially at thermal equilibrium. Throughout the paper, the subscript "0" will denote properties referring to this situation. Let $p_{e q, 0}(\mathbf{x}) \propto e^{-V_{0}(\mathbf{X})}$ be the distribution of the microstates where $V_{0}(\mathbf{x})$ is the configurational energy of the unperturbed system. With reference to such an equilibrium state, the free energy is related to the configurational canonical partition function via $A_{0}=-\ln \int d \mathbf{x} e^{-V_{0}(\mathbf{X})}$ (except for an immaterial addend which is constant at a fixed temperature). Now suppose that the external agent starts to modulate the internal energy of the system so that $V_{0}(\mathbf{x})$ switches to $V(\mathbf{x}, t)$ for $t \geq 0$ (but without discontinuity, that is $\left.V(\mathbf{x}, 0)=V_{0}(\mathbf{x})\right)$. During the transformation, the system is out-of-equilibrium. However, at any time $t$, reference can be made to the "underlying" equilibrium state which corresponds 
to the Boltzmann distribution $p_{e q, t}(\mathbf{x}) \propto e^{-V_{t}(\mathbf{x})}$ with $V_{t}(\mathbf{x}) \equiv V(\mathbf{x}, t)$. The thermodynamic state-functions, for example $A_{t}$, are defined for this underlying equilibrium state.

From this perspective, what emerged from the pionieristic works of Jarzynski [17, 18] is that the familiar expressions of the Second Principle of thermodynamics of macroscopic systems still hold at the molecular scale but only on average. In particular, the Clausius inequality for systems transformed at a fixed temperature becomes $\bar{w}-\Delta A>0$, where $\Delta A=A_{t}-A_{0}$ is the free energy variation between the (underlying) equilibrium state reached at time $t$ and the initial equilibrium state, and $\bar{w}$ is the average work done until that time $t$; the average is taken over the statistical ensemble of trajectories generated under the same conditions, i.e., $i)$ initial microstates $\mathbf{x}(0)_{t r}$ sampled from the distribution $p_{e q, 0}(\mathbf{x})$ and $\left.i i\right)$ same transformation protocol. For the sake of notation, in the following the dependence of $\bar{w}$ and $\Delta A$ on $t$ is not indicated unless it is needed for clarity. The difference $\bar{w}_{\text {diss }}=\bar{w}-\Delta A$, always positive, corresponds to the average energy dissipation which can be intuitively interpreted as the amount of energy "wasted" due to the inevitable "lag" between the system's response and the perturbing agent when the transformation is performed in a finite time (i.e., in the jargon of thermodynamics, when the transformation is "irreversible" in contrast with a "reversible" or "quasi-static" one). Such a connection has been made explicit [19] showing that, at any time $t, \bar{w}_{\text {diss }}$ sets an upper limit to the deviation, measured in terms of relative entropy [20], between the actual out-of-equilibrium distribution $p(\mathbf{x}, t)$ and the underlying distribution $p_{e q, t}(\mathbf{x})$.

Let us now specify the above quite general remarks in regard to cyclic perturbations. An abstract representation of the problem is given in Figure 1a. Here we consider the case of $V(\mathbf{x}, t)$ modulated with a period $\tau$. Namely, the modulation is introduced through the energy perturbation $V_{1}(\mathbf{x}, t)$ such that

$$
V(\mathbf{x}, t)=V_{0}(\mathbf{x})+V_{1}(\mathbf{x}, t) \quad, \quad V_{1}\left(\mathbf{x}, n_{c} \tau\right)=V_{1}(\mathbf{x}, 0)=0 \text { all } \mathbf{x}
$$

where $n_{c}$ is any integer number of cycles. In all generality, for each $\mathbf{x}$ the time-modulation may feature several Fourier components with frequencies multiples of the fundamental one $\omega=2 \pi / \tau$. Since the system retrieves the same (underlying) equilibrium state after the completion of each cycle, the variation of any thermodynamic state function is null after a generic number of cycles. In particular, $\Delta A=0$ implies that $\bar{w}_{\text {diss }} \equiv \bar{w}$ after an integer number of cycles. With reference to the amount of work $\bar{w}_{\text {diss }, n_{c}}$ which is dissipated on average during the $n_{c}$-th cycle of the perturbation, we have

$$
\bar{w}_{\text {diss }, n_{c}} \equiv \bar{w}_{n_{c}}
$$

Our main focus will be on the limit value

$$
\bar{w}_{\text {diss }}^{\infty}=\lim _{n_{c} \rightarrow \infty} \bar{w}_{\text {diss }, n_{c}}
$$


As the number of performed cycles increases, the non-equilibrium distribution on the $\mathbf{x}$ variables tends to evolve coherently in a steady-state with the external perturbation[10] and the limit in Eq. 4 is indeed asymptotically reached (as will be shown). Since $\bar{w}_{\text {diss }}>0$ after the completion of any number of cycles, it can be deduced that the contribution to $\bar{w}_{\text {diss }}$ per cycle in the asymptotic limit must be positive, i.e., $\bar{w}_{\text {diss }}^{\infty}>0$.

Note that the quantity $\bar{w}_{\text {diss }}^{\infty}$ also corresponds to the average environmental entropy production (in $k_{B}$ units), per cycle, in the steady-state condition. To see this one can start from the general relation $\bar{w}-\Delta A \equiv \bar{w}_{\text {diss }}=\Delta S+\overline{\Delta S}_{\text {ext }}$ (see the discussion in section 7 of ref. [3] concerning the entropy production topic) where $\Delta S$ is the entropy variation of the systems (between the final "underlying" equilibrium state and the initial equilibrium state) and $\overline{\Delta S}_{\text {ext }}$ is the average entropy variation of the environment as consequence of the finite-time transformation protocol (clearly, $\overline{\Delta S}_{\text {ext }}$ also includes the final exchange of heat between system and environment which occurs in the relaxation phase after the action of the protocol is concluded); to interpret the relations given above, it is important to recall that the energy quantities are expressed in $k_{B} T$ units and the entropy in $k_{B}$ units. In our specific case, since $\Delta S=0$ after a generic number of cycles, it follows that $\bar{w}_{\text {diss }}^{\infty}$ gives the average contribution to the entropy produced in the environment (in $k_{B}$ units) due to the realization of each cycle at the steady-state.

\section{FIGURE 1}

At this point, some remarks can be made about the physical nature of the perturbing agent. Such an agent may be 1) an external device able to "tether" itself to one or more "exposed features" of the system and to modulate its (their) state according to a prescribed cyclic time schedule; for each actual state(s) of this (these) parameter(s), the energetics of the system is different, hence a time modulation of $V(\mathbf{x}, t)$ is induced. As an alternative, the perturbing agent may be 2) an external field which evolves periodically; the coupling between that type of field and some property of the system gives rise to the perturbed energy function $V(\mathbf{x}, t)$. Finally, the perturbing agent may be 3 ) an ensemble of physico-chemical events able to change the structural properties of the system in such a way that the parameters which enter the functional form of $V(\mathbf{x}, t)$ evolve periodically; for example a network of chemical reactions which may generate a periodic flow of chemicals through the systems, so that the actual concentrations of the species determine the parameters of $V(\mathbf{x}, t)$.[21] In case 1) there is a continuous "tuning" of the external device during the system's evolution, hence the dissipated energy can be interpreted as the "extra amount" of energy which is "put into play" (ceded or acquired) to guarantee that the time-schedule along the actual trajectory 
$\mathbf{x}_{t r}(t)$ is realized. In cases 2) and 3) this kind of tuning is missing and nothing more can be said beyond the fact that the dissipation is associated to the lag between the system's response and the external agent. Since $\bar{w}_{\text {diss }}^{\infty}$ is positive, in case 1 ) we can talk about the average "energy cost" from the point of view of the external device, while in cases 2) and 3 ) it would make more sense to refer to the average "energy wasted" given that it is energy withdrawn from the external agent but not used to perform work.

Clearly, $\bar{w}_{\text {diss }}^{\infty}$ (and each of the terms $\bar{w}_{\text {diss }, n_{c}}$ as well) depends on the specific kind of energy perturbation and on the dynamic response of the system. In practice, the system responds to the perturbation by adopting its intrinsic modes/rates with a weight which depends on the specific form of $V(\mathbf{x}, t)$. The way that is pursued here to specify unambiguously intrinsic and time-invariant modes and rates is to refer to the thermal fluctuations of the unperturbed system. Namely, we will identify the modes and the associated rates as, respectively, the eigenfunctions and the eigenvalues of the symmetrized Smoluchowski operator which describes the evolution of the probability distribution $p_{0}(\mathbf{x}, t)$ for the unperturbed system, with $p_{0}(\mathbf{x}, t) \rightarrow p_{e q, 0}(\mathbf{x})$ for any initial condition. This choice is adequate if the magnitude of the perturbation is small enough so that these "reference" modes/rates can still keep this privileged status even in an out-of-equilibrium situation.

Our objective is to explore the interrelations depicted in Figure 1b. For that purpose, we derived an approximate although explicit expression for $\bar{w}_{\text {diss }}^{\infty}(\omega)$ which links all the physical ingredients in a transparent way. Such a relation is valid if the perturbations are week, since it is obtained through an appropriate perturbative treatment till first order in the strength of the energy perturbation. In particular, we will investigate the low- and high-frequency limits of $\bar{w}_{\text {diss }}^{\infty}(\omega)$ showing that the average dissipation tends to vanish; then we inspect the location of the maxima in terms of closeness of $\omega$ to some intrinsic fluctuation rates at equilibrium. In this regard, it is possible to refer to "stochastic resonance" [22] since the match between external frequency and fluctuation rates can enhance a particular response which, in this case, is the amount of energy dissipated per cycle[6, 7].

To test the effectiveness of the analytical approximation, we performed model calculations on a simple uni-dimensional case model (a single degree of freedom $x$ ) constituted by a "particle on a ring" which fluctuates in a bi-stable potential $V_{0}(x)$ with equivalent wells and perturbed in two ways: in one, the energy barrier is periodically modulated and $V(x, t)$ keeps a symmetric profile; in the other, the whole energy profile is changed in such a way that the initial even symmetry is broken. The outcomes from application of the analytical approximation are compared with the exact results obtained by the numerical solution of the non-stationary Fokker-Planck-Smoluchowski equation. A bi-stable case was chosen because of its wide employment as a benchmark model both in studies on fluctuations at equilibrium (see for example ref. [23] for overdamped dynamics) and energy dissipation under driven 
transformations [6,7]. Although these studies on cyclic perturbations were performed on different kinds of bi-stable potentials and with different boundary conditions, we anticipate that there is a qualitative accord with some of the features that emerge here.

The paper is structured as follows. The next section outlines the theoretical framework: the stationary and non-stationary Fokker-Planck-Smoluchowski equations adopted to model the fluctuations in the diffusive regime, the general form of energy perturbation, and the definition of average energy dissipation per cycle. In section III we present the analytical approximation of $\bar{w}_{\text {diss }}^{\infty}$ and discuss its physical implication; compact expressions of $\bar{w}_{\text {diss }}^{\infty}$ and related quantities are also given (derivations are provided in the Appendix A). Section IV is devoted to model calculations: presentation of the case model, computational details and outcomes. In Section V we draw the main conclusions and outline some speculative arguments for future investigations. Appendix B contains a supplementary inspection on the statistical properties of $w_{\text {diss }}^{\infty}$. Some relevant remarks are provided in the notes.

\section{THEORETICAL FRAMEWORK}

\section{A. Fluctuations at equilibrium and under perturbation}

Let $\mathbf{x}$ be a suitable set of continuous variables which specify the configuration of the system and whose dynamics is a Markov process in the overdamped regime of motion. Let us first focus on the condition of unperturbed system at thermal equilibrium. We recall that the subscript "0" denotes properties referring to such a situation. At equilibrium, the probability distribution takes the Boltzmann form

$$
p_{e q, 0}(\mathbf{x})=e^{-V_{0}(\mathbf{x})} / \int d \mathbf{x} e^{-V_{0}(\mathbf{x})}
$$

For general functions (possibly time-dependent) of the system's configuration, the equilibrium ensemble average will be indicated with the notation

$$
\langle f(\mathbf{x}, t)\rangle_{0} \equiv \int d \mathbf{x} f(\mathbf{x}, t) p_{e q, 0}(\mathbf{x})
$$

The probability distribution $p_{0}(\mathbf{x}, t)$ evolves according to the stationary Fokker-Planck equation

$$
\frac{\partial p_{0}(\mathbf{x}, t)}{\partial t}=-\hat{\Gamma}_{0} p_{0}(\mathbf{x}, t) \quad, \quad \hat{\Gamma}_{0}=-\frac{\partial}{\partial \mathbf{x}}^{T} \mathbf{D}(\mathbf{x}) e^{-V_{0}(\mathbf{x})} \frac{\partial}{\partial \mathbf{x}} e^{+V_{0}(\mathbf{x})}
$$

where $\hat{\Gamma}_{0}$ is the Smoluchowski operator for overdamped dynamics, with $\partial / \partial \mathbf{x}$ the gradient operator on the $\mathbf{x}$ variables ("T" stands for the transposed array) and $\mathbf{D}(\mathbf{x})$ the diffusion matrix which may generally depend on the system's configuration. In the long-time limit, and regardless of the initial condition $p_{0}(\mathbf{x}, 0)$, the distribution $p_{0}(\mathbf{x}, t)$ tends to $p_{e q, 0}(\mathbf{x})$. 
In order to deal with an hermitian operator, the following "symmetrization" is applied:

$$
\tilde{\Gamma}_{0}=p_{e q, 0}(\mathbf{x})^{-1 / 2} \hat{\Gamma}_{0} p_{e q, 0}(\mathbf{x})^{1 / 2}
$$

The eigenfunctions $\phi_{n}(\mathbf{x})$ (generally complex-valued) of $\tilde{\Gamma}_{0}$, each associated to an eigenvalue $\lambda_{n}$ (real-valued), form an ortho-normal basis which can be employed to expand functions of the $\mathbf{x}$ variables:

$$
\tilde{\Gamma}_{0} \phi_{n}(\mathbf{x})=\lambda_{n} \phi_{n}(\mathbf{x}) \quad, \quad \int d \mathbf{x} \phi_{n}(\mathbf{x})^{*} \phi_{n^{\prime}}(\mathbf{x})=\delta_{n, n^{\prime}}
$$

In particular,

$$
\lambda_{0}=0, \quad \lambda_{n \geq 1}>0 \quad, \quad \phi_{n}(\mathbf{x})=g_{n}(\mathbf{x}) p_{e q, 0}(\mathbf{x})^{1 / 2}
$$

with the functions $g_{n}(\mathbf{x})$ such that

$$
g_{0}(\mathbf{x})=1 \quad, \quad\left\langle g_{n}(\mathbf{x})^{*} g_{n^{\prime}}(\mathbf{x})\right\rangle_{0}=\delta_{n, n^{\prime}}
$$

where the notation in Eq. 6 has been used and $\delta$ stands for Kronecker's Delta function. Note that the symmetrization in Eq. 9 only changes the eigenfunctions (those of $\hat{\Gamma}_{0}$ are the $\phi_{n}(\mathbf{x})$ multiplied by $\left.p_{e q, 0}(\mathbf{x})^{1 / 2}\right)$ but it does not alter the eigenvalues. The $\lambda_{n}$ are interpreted as intrinsic rates of fluctuations, that is, as rates of relaxation of the $p_{0}(\mathbf{x}, t)$ landscape (towards $\left.p_{e q, 0}(\mathbf{x})\right)$ over each "independent mode"; the explicit integration of Eq. 7 indeed yields

$$
p_{0}(\mathbf{x}, t)=p_{e q, 0}(\mathbf{x})\left\{1+\sum_{n \geq 1} c_{n} g_{n}(\mathbf{x}) e^{-\lambda_{n} t}\right\} \quad, \quad c_{n}=\int d \mathbf{x} g_{n}(\mathbf{x})^{*} p_{0}(\mathbf{x}, 0)
$$

The functions $g_{n}(\mathbf{x})$, which are eigenfunctions of the adjoint operator $\hat{\Gamma}_{0}^{\dagger}$, as can be verified directly, will play an important role in the decomposition of the energy perturbation. We would like to point out that the usefulness of these functions as target collective coordinates in the non-linear mapping known as "diffusion maps", a tool which is applied in the dimensional reduction of complex fluctuating systems, has recently been recognized.[24, 25]

Under application of the perturbation $V_{1}(\mathbf{x}, t)$, the probability distribution, now denoted as $p(\mathbf{x}, t)$, evolves according to the non-stationary Fokker-Planck-Smoluchowski equation

$$
\frac{\partial p(\mathbf{x}, t)}{\partial t}=-\hat{\Gamma}(t) p(\mathbf{x}, t) \quad, \quad \hat{\Gamma}(t)=-\frac{\partial}{\partial \mathbf{x}}^{T} \mathbf{D}(\mathbf{x}) e^{-V(\mathbf{x}, t)} \frac{\partial}{\partial \mathbf{x}} e^{+V(\mathbf{x}, t)}
$$

where we have assumed that the diffusion matrix is unaltered by the perturbation, i. e., that the perturbation does not affect the "hydrodynamics" of the system but only its energetics. The time-dependence of the evolution operator now prevents an "exponentialization" as in Eq. 12 and the solution $p(\mathbf{x}, t)$ must be generally achieved numerically or by applying sensible approximations. 


\section{B. Form of the cyclic energy perturbation}

The energy perturbation $V_{1}(\mathbf{x}, t)$ in Eq. 2 is decomposed in a Fourier series,

$$
V_{1}(\mathbf{x}, t)=\sum_{N=-\infty}^{+\infty} v(N, \mathbf{x}) e^{i N \omega t} \quad, \quad \omega=\frac{2 \pi}{\tau}
$$

where the Fourier components are obtained by the inversion formula

$$
v(N, \mathbf{x})=\frac{1}{\tau} \int_{0}^{\tau} d t V_{1}(\mathbf{x}, t) e^{-i N \omega t}
$$

The relation $v(-N, \mathbf{x})=v(N, \mathbf{x})^{*}$ follows by the fact that $V_{1}(\mathbf{x}, t)$ is a real-valued function. Moreover, $\sum_{N=-\infty}^{+\infty} v(N, \mathbf{x})=0$ since $V_{1}(\mathbf{x}, m \tau)=0$ for any integer $m \geq 0$ and for any configuration $\mathbf{x}$.

The Fourier components are then decomposed on the set of functions $g_{n}(\mathbf{x})$ defined in Eq. 10:

$$
v(N, \mathbf{x})=\sum_{n} v_{n}(N) g_{n}(\mathbf{x})
$$

Such a decomposition is justified in consideration of the fact that $v(N, \mathbf{x}) p_{e q, 0}(\mathbf{x})^{1 / 2}$ can be first expanded onto the ortho-normal basis of the $\phi_{n}(\mathbf{x})$ functions: $v(N, \mathbf{x}) p_{e q, 0}(\mathbf{x})^{1 / 2}=$ $\sum_{n} v_{n}(N) \phi_{n}(\mathbf{x})$. Then, by multiplying both members by $p_{e q, 0}(\mathbf{x})^{-1 / 2}$, and recalling that $\phi_{n}(\mathbf{x})=g_{n}(\mathbf{x}) p_{e q, 0}(\mathbf{x})^{1 / 2}$, Eq. 16 follows. The coefficients $v_{n}(N)$ are given by $v_{n}(N)=$ $\int d \mathbf{x} \phi_{n}(\mathbf{x})^{*} v(N, \mathbf{x}) p_{e q, 0}(\mathbf{x})^{1 / 2} \equiv\left\langle g_{n}(\mathbf{x})^{*} v(N, \mathbf{x})\right\rangle_{0}$. By inserting Eq. 15 in this integral, it follows

$$
v_{n}(N)=\frac{1}{\tau} \int_{0}^{\tau} d t\left\langle g_{n}(\mathbf{x})^{*} V_{1}(\mathbf{x}, t)\right\rangle_{0} e^{-i \omega_{N} t}
$$

Note that although the period $\tau$ enters Eq. 17, the coefficients $v_{n}(N)$ do not depend on it. $[26]$

\section{Average energy dissipation per cycle and its asymptotic limit}

Let us consider a stochastic trajectory $\mathbf{x}(t)_{t r}$ under the action of the perturbation. By integrating the infinitesimal work amount given in Eq. 1, the net work done between two instants $t_{1}$ and $t_{2}$ is

$$
w_{t r}\left(t_{1}, t_{2}\right)=\left.\int_{t_{1}}^{t_{2}} d t \frac{\partial V(\mathbf{x}, t)}{\partial t}\right|_{\mathbf{x}=\mathbf{x}(t)_{t r}}
$$

By taking the average over an infinite number of trajectories, each starting from a configuration "picked" from the same initial distribution (recall that $p(\mathbf{x}, 0)=p_{e q, 0}(\mathbf{x})$ in the present 
case), we attain the average work $\bar{w}\left(t_{1}, t_{2}\right)$. This operation can be replaced by the equivalent average over the time-dependent probability distribution, that is

$$
\bar{w}\left(t_{1}, t_{2}\right)=\int_{t_{1}}^{t_{2}} d t \int d \mathbf{x} \frac{\partial V(\mathbf{x}, t)}{\partial t} p(\mathbf{x}, t)
$$

With these positions, the average work done during the $n_{c^{-}}$th cycle $\bar{w}_{n_{c}}$, is given by Eq. 19 with $t_{1}=t_{n_{c}-1}$ and $t_{2}=t_{n_{c}}$.

By recalling that $\bar{w}_{n_{c}}$ coincides with the average dissipated energy per cycle, $\bar{w}_{\text {diss }, n_{c}}$ (see Eq. 3), it follows

$$
\bar{w}_{\text {diss }, n_{c}}=\int_{t_{n_{c}-1}}^{t_{n_{c}}} d t \int d \mathbf{x} \frac{\partial V(\mathbf{x}, t)}{\partial t} p(\mathbf{x}, t)
$$

The asymptotic value $\bar{w}_{\text {diss }}^{\infty}$ is then obtained as the limit of the succession of the $\bar{w}_{\text {diss }, n_{c}}$ values (see Eq. 4).

The integral in Eq. 20 can be evaluated as an average over a sufficiently large sample of simulated trajectories or by solving numerically the non-stationary Fokker-PlanckSmoluchowski equation to attain $p(\mathbf{x}, t)$. In the following we will employ both strategies for calculations on simple model systems. The latter route, however, will be the preferred one to obtain the reference profiles of $\bar{w}_{\text {diss }}^{\infty}$ vs. $\omega$ to be used to assess the quality of the analytical approximation presented in Section III.

\section{ANALYTICAL RESULTS}

\section{A. Main outcomes}

An analytical approximation of $\bar{w}_{\text {diss }}^{\infty}$ is obtained here under the assumption that the magnitude of the perturbation is small enough that the system is only slightly out of thermal equilibrium. In Section III B we will provide the algebraic elaboration based on such an assumption. It is demonstrated (see Eqs. 39 - 41) that the sequence of $\bar{w}_{\text {diss }, n_{c}}$ does indeed converge to an asymptotic value. Such a trend is assured by the positivity of the eigenvalues $\lambda_{n \geq 1}$ of the reference Smoluchowski operator $\hat{\Gamma}_{0}$. Specifically, the following explicit formula is attained

$$
\bar{w}_{\text {diss }}^{\infty}(\omega) \simeq 4 \pi \sum_{n \geq 1} \sum_{N \geq 1} \frac{Q(n, N)}{\lambda_{n} / \omega_{N}+\omega_{N} / \lambda_{n}} \quad, \quad \omega_{N}=N \omega \quad, \quad Q(n, N)=N\left|v_{n}(N)\right|^{2}
$$

where the factors $v_{n}(N)$ are given in Eq. 17. In practice, $\bar{w}_{\text {diss }}^{\infty}(\omega)$ is found to be a superposition of functions $\mathcal{D}_{\lambda_{n}}\left(\omega_{N}\right):=\left(\lambda_{n} / \omega_{N}+\omega_{N} / \lambda_{n}\right)^{-1}$ [27] which take the same maximum value $1 / 2$ at the "resonance" frequency $\omega_{N}=\lambda_{n}$. Each of these functions is weighted by a $\omega$-independent factor $Q(n, N)$ whose magnitude depends on the projection of the $N$-th 
Fourier component of the energy perturbation (see Eq. 14) on the $n$-th mode of fluctuation (according to Eq. 16). The multiplication of $\bar{w}_{\text {diss }}^{\infty}(\omega)$ by $\tau^{-1}=\omega / 2 \pi$ then yields the average dissipation rate. The symbol " $\simeq$ ", here and throughout the text, means that the expression on the right side is an approximation which is acceptable, within a required tolerance, if specific conditions are fulfilled. In this case, it is essential to keep in mind that Eq. 21 is derived by means of a first-order perturbative treatment in the strength of the energy perturbation. All deductions and further elaborated results rely, implicitly, on the applicability of this linear approximation. Unfortunately, as is common in linear-response-like methodologies, the error committed remains undetermined.

The low- and high-frequency limits of $\bar{w}_{\text {diss }}^{\infty}(\omega)$ are readily derived from Eq. 21:

$$
\begin{aligned}
& \text { low } \omega: \bar{w}_{\text {diss }}^{\infty} \simeq c_{1} \omega, \quad c_{1}=4 \pi \sum_{n \geq 1}\left(\lambda_{n}\right)^{-1} \sum_{N \geq 1}\left|N v_{n}(N)\right|^{2} \\
& \text { high } \omega: \bar{w}_{\text {diss }}^{\infty} \simeq c_{2} / \omega, \quad c_{2}=4 \pi \sum_{n \geq 1} \lambda_{n} \sum_{N \geq 1}\left|v_{n}(N)\right|^{2}
\end{aligned}
$$

Clearly, equations 22 are meaningful only if the summatories that express the coefficients $c_{1}$ and $c_{2}$ converge to finite values. From a mathematical viewpoint, the low-frequency approximation holds if $\omega \ll \lambda_{n} / N$ for each pair $(n, N)$. If the eigenvalues are listed in ascending order, the condition becomes $\omega \ll \lambda_{1} / N^{*}$ where $N^{*}=\arg \max _{N}\left\{\max _{n} Q(n, N)\right\}$. Similarly, the high-frequency approximation holds if $\omega \gg \lambda_{n} / N$ for each pair $(n, N)$; this requires $\omega \gg \lambda_{n^{*}}$ where $n^{*}=\arg \max _{n}\left\{\max _{N} Q(n, N)\right\}$. Thus, the validity of Eqs. 22 relies on the possibility of identifying the integers $n^{*}$ and $N^{*}$ for the specific kind of perturbation. However, the validity of Eqs. 22 is expected in practice, at least because they agree with the intuitive picture that the average dissipation per cycle tends to vanish if the perturbation switches so quickly that the system is essentially "transparent" to it, or if the perturbation evolves so slowly that the system is transformed in a quasi-static way. By turning to the rate (time-averaged over one period) of average energy dissipation, that is the quantity $\omega \bar{w}_{\text {diss }}^{\infty}(\omega) / 2 \pi$, Eqs. 22 predict that the rate grows quadratically with $\omega$ in the low-frequency range, while it tends to a finite value as $\omega \rightarrow \infty(\tau \rightarrow 0)$. Interestingly, the limit of finite average dissipation rate when $\tau \rightarrow 0$ was recently found [28] also for overdamped fluctuations in a uni-dimensional harmonic potential subjected to forward/backward stochastic switch of its center between two values; in that study, the switches are modeled as a Poisson process and $\tau$ is related to the inverse of the switching rate.

In a double-logarithmic scale, the relations in Eqs. 22 give straight lines with slopes +1 and -1 . The crossing of these lines occurs at the frequency $\sqrt{c_{2} / c_{1}}$. By means of a majorization of Eq. 21 it is easy to see that the graph of $\bar{w}_{\text {diss }}^{\infty}(\omega)$ entirely lies underneath these lines. In particular, the following upper bound can be given for the maximum average 
dissipation:

$$
\max _{\omega}\left\{\bar{w}_{\text {diss }}^{\infty}(\omega)\right\}<\sqrt{c_{1} c_{2}}
$$

We stress again that this inequality relies on the approximation Eq. 21, hence it is valid only for small enough energy perturbations.

Now let us consider the eigenvalues $\lambda_{n}$ listed in ascending order, and suppose to be in the situation in which they can be divided into sets well separated one from the other. Namely, let $s$ be an index which labels these sets, with $\lambda_{\max , s}=\max _{n \in \operatorname{set} s}\left\{\lambda_{n}\right\}$ and $\lambda_{\min , s}=$ $\min _{n \in \operatorname{set} s}\left\{\lambda_{n}\right\}$ the upper and lower values in the $s$-th set; a criterion for the separation of the $s$-th set by the $(s-1)$-th and $(s+1)$-th ones might be that $\lambda_{\min , s+1} / \lambda_{\max , s} \geq r_{\text {gap }}$ and $\lambda_{\text {min }, s} / \lambda_{\text {max }, s-1} \geq r_{\text {gap }}$ with $r_{\text {gap }} \gg 1$ a chosen threshold ratio used to detect the existence of gaps. Then we can write $\bar{w}_{\text {diss }}^{\infty}(\omega)=\sum_{s} \bar{w}_{\text {diss }, s}^{\infty}(\omega)$, where the partial contributions $\bar{w}_{\text {diss }, s}^{\infty}(\omega)$ are given by Eq. 21 only with $n \in$ set $s$. Here below the frequency profile of $\bar{w}_{\text {diss }, s}^{\infty}(\omega)$ will be called "s-th band". If we focus on the frequency range $\lambda_{\max , s} \ll \omega \ll \lambda_{\min , s+1}$, it might be the case that the following conditions are met: $i)$ the high-frequency and low-frequency approximations are applicable, respectively, to the $s$ and $(s+1)$ bands; ii) at each frequency in that range, the bands $s^{\prime}<s$ and $s^{\prime}>s+1$ give a negligible global contribution to $\bar{w}_{\text {diss }}^{\infty}(\omega)$; iii) the bands $s$ and $(s+1)$ are resolved one from the other, at least partially. Similarly, the symmetrical behavior might be observed in the frequency range $\lambda_{\max , s-1} \ll \omega \ll \lambda_{\min , s}$. If these conditions are met, the contribution to the average dissipation due to the $s$-th set of modes/eigenvalues is separated, in terms of the appearance of a resolved band, from the contribution of the rest of the modes. This feature can indeed be observed depending on the kind of external perturbation which affects the weight factors in Eq. 21. In other words, if the external perturbation is suitably tuned, possible gaps in the spectrum of the internal fluctuation rates are revealed by resolved bands in the $\bar{w}_{\text {diss }}^{\infty}(\omega)$ profile. The calculations presented in Section IV will illustrate this feature in a simple case.

\section{B. Derivation of Eq. 21}

Let us start by inserting $V(\mathbf{x}, t)=V_{0}(\mathbf{x})+V_{1}(\mathbf{x}, t)$ into the Smoluchowski operator $\hat{\Gamma}(t)$ in Eq. 13. A few steps lead to the following partition

$$
\hat{\Gamma}(t)=\hat{\Gamma}_{0}+\hat{S}(t)
$$

with

$$
\hat{\Gamma}_{0}=-\frac{\partial}{\partial \mathbf{x}}^{T} \mathbf{D}(\mathbf{x}) e^{-V_{0}(\mathbf{x})} \frac{\partial}{\partial \mathbf{x}} e^{+V_{0}(\mathbf{x})} \quad, \quad \hat{S}(t)=-\frac{\partial}{\partial \mathbf{x}}^{T} \mathbf{D}(\mathbf{x}) \frac{\partial V_{1}(\mathbf{x}, t)}{\partial \mathbf{x}}
$$

The formal integration of the Fokker-Planck-Smoluchowski equation yields

$$
p(\mathbf{x}, t)=p_{e q, 0}(\mathbf{x})-\int_{0}^{t} d t^{\prime} e^{-\left(t-t^{\prime}\right) \hat{\Gamma}_{0}} \hat{S}\left(t^{\prime}\right) p\left(\mathbf{x}, t^{\prime}\right)
$$


where it has been used $p(\mathbf{x}, 0)=p_{e q, 0}(\mathbf{x})$ since the system is initially, by assumption, at thermal equilibrium. By using recursively Eq. 26 in the integrand, the following form for the non-equilibrium distribution is attained:

$$
\begin{aligned}
p(\mathbf{x}, t)=p_{e q, 0}(\mathbf{x}) & +\sum_{k=1}^{\infty}(-1)^{k} \int_{0}^{t} d t^{(1)} \int_{0}^{t^{(1)}} d t^{(2)} \ldots \\
& \cdots \int_{0}^{t^{(k-1)}} d t^{(k)} \hat{O}\left(t, t^{(1)}\right) \hat{O}\left(t^{(1)}, t^{(2)}\right) \cdots \hat{O}\left(t^{(k-1)}, t^{(k)}\right) p_{e q, 0}(\mathbf{x})
\end{aligned}
$$

where, for the sake of notation, we have introduced the following operator:

$$
\hat{O}\left(t, t^{\prime}\right):=e^{-\left(t-t^{\prime}\right) \hat{\Gamma}_{0}} \hat{S}\left(t^{\prime}\right)
$$

Each operator $\hat{O}$ brings a contribution proportional to $V_{1}$, hence Eq. 27 can be seen as an expansion into power series of the magnitude of the energy perturbation. For small perturbations, the truncation up to the first relevant term $(k=1)$ yields

$$
p(\mathbf{x}, t) \simeq p_{e q, 0}(\mathbf{x})-\int_{0}^{t} d t^{\prime} e^{-\left(t-t^{\prime}\right) \hat{\Gamma}_{0}} \hat{S}\left(t^{\prime}\right) p_{e q, 0}(\mathbf{x})
$$

Let us now consider the identity

$$
\hat{S}(t) p_{e q, 0}(\mathbf{x}) \equiv \hat{\Gamma}_{0} p_{e q, 0}(\mathbf{x}) V_{1}(\mathbf{x}, t)
$$

Application of such a relation into the integrand in Eq. 29 yields $e^{-\left(t-t^{\prime}\right) \hat{\Gamma}_{0}} \hat{S}\left(t^{\prime}\right) p_{e q, 0}(\mathbf{x})=$ $e^{-\left(t-t^{\prime}\right) \hat{\Gamma}_{0}} \hat{\Gamma}_{0} p_{e q, 0}(\mathbf{x}) V_{1}\left(\mathbf{x}, t^{\prime}\right) \equiv \partial e^{-\left(t-t^{\prime}\right) \hat{\Gamma}_{0}} / \partial t^{\prime} p_{e q, 0}(\mathbf{x}) V_{1}\left(\mathbf{x}, t^{\prime}\right)$. Integration by parts with the use of the condition $V_{1}(\mathbf{x}, 0)=0$ leads to

$$
p(\mathbf{x}, t) \simeq p_{e q, 0}(\mathbf{x})-p_{e q, 0}(\mathbf{x}) V_{1}(\mathbf{x}, t)+\int_{0}^{t} d t^{\prime} e^{-\left(t-t^{\prime}\right) \hat{\Gamma}_{0}} p_{e q, 0}(\mathbf{x}) \frac{\partial V_{1}\left(\mathbf{x}, t^{\prime}\right)}{\partial t^{\prime}}
$$

Such an approximation is now used to compute the average work done until time $t$ according to Eq. 19:

$$
\bar{w}(0, t)=\int_{0}^{t} d t^{\prime} \int d \mathbf{x} \frac{\partial V\left(\mathbf{x}, t^{\prime}\right)}{\partial t^{\prime}} p\left(\mathbf{x}, t^{\prime}\right) \equiv \int_{0}^{t} d t^{\prime} \int d \mathbf{x} \frac{\partial V_{1}\left(\mathbf{x}, t^{\prime}\right)}{\partial t^{\prime}} p\left(\mathbf{x}, t^{\prime}\right)
$$

By inserting Eq. 31 in Eq. 32, and turning to the symmetrized Smoluchowski operator defined in Eq. 8, we obtain

$$
\begin{aligned}
\bar{w}(0, t) & \simeq\left\langle V_{1}(t)\right\rangle_{0}-\frac{1}{2}\left\langle V_{1}(t)^{2}\right\rangle_{0} \\
& +\int_{0}^{t} d t^{\prime} \int_{0}^{t^{\prime}} d t^{\prime \prime} \int d \mathbf{x} \frac{\partial V_{1}\left(\mathbf{x}, t^{\prime}\right)}{\partial t^{\prime}} p_{e q, 0}(\mathbf{x})^{1 / 2} e^{-\left(t^{\prime}-t^{\prime \prime}\right) \tilde{\Gamma}_{0}} p_{e q, 0}(\mathbf{x})^{1 / 2} \frac{\partial V_{1}\left(\mathbf{x}, t^{\prime \prime}\right)}{\partial t^{\prime \prime}}
\end{aligned}
$$


where the notation in Eq. 6 has been adopted to indicate the equilibrium averages, and where it has been considered that $\int_{0}^{t} d t^{\prime}\left\langle\partial V_{1}\left(\mathbf{x}, t^{\prime}\right) / \partial t^{\prime}\right\rangle_{0}=\left\langle V_{1}(\mathbf{x}, t)\right\rangle_{0}$ and $\int_{0}^{t} d t^{\prime}\left\langle V_{1}\left(\mathbf{x}, t^{\prime}\right) \partial V_{1}\left(\mathbf{x}, t^{\prime}\right) / \partial t^{\prime}\right\rangle_{0}=\int_{0}^{t} d t^{\prime}\left\langle\partial V_{1}\left(\mathbf{x}, t^{\prime}\right)^{2} / \partial t^{\prime}\right\rangle_{0} / 2=\left\langle V_{1}(\mathbf{x}, t)^{2}\right\rangle_{0} / 2$ since $V_{1}(\mathbf{x}, 0)=0$. From the Fourier decomposition in Eq. 14 it follows

$$
\frac{\partial V_{1}(\mathbf{x}, t)}{\partial t} p_{e q, 0}(\mathbf{x})^{1 / 2}=i \omega \sum_{N}\left[v(N, \mathbf{x}) p_{e q, 0}(\mathbf{x})^{1 / 2}\right] N e^{i N \omega t}
$$

The factors in the square brackets on the right-hand side are now decomposed on the basis of the eigenfunction $\phi_{n}(\mathbf{x})$ of the operator $\tilde{\Gamma}_{0}$ :

$$
v(N, \mathbf{x}) p_{e q, 0}(\mathbf{x})^{1 / 2}=\sum_{n} v_{n}(N) \phi_{n}(\mathbf{x})
$$

where the coefficients $v_{n}(N)$ are expressed in Eq. 16. The use of Eqs. 34-35 in Eq. 33 leads to attain, after some algebraic steps omitted here, the following compact expression for the average work:

$$
\bar{w}(0, t) \simeq\left\langle V_{1}(t)\right\rangle_{0}-\frac{1}{2}\left\langle V_{1}(t)^{2}\right\rangle_{0}+\sum_{n} \sum_{N, N^{\prime}} \alpha_{n}\left(t, N, N^{\prime}\right)
$$

with

$$
\alpha_{n}\left(t, N, N^{\prime}\right)=-\omega^{2} \frac{N N^{\prime} v_{n}(N) v_{n}\left(N^{\prime}\right)}{\lambda_{n}+i \omega N^{\prime}}\left[\frac{e^{i \omega\left(N+N^{\prime}\right) t}-1}{i \omega\left(N+N^{\prime}\right)}+\frac{e^{-\left(\lambda_{n}-i \omega N\right) t}-1}{\lambda_{n}-i \omega N}\right]
$$

where the $\lambda_{n}$ are the eigenvalues of the Smoluchowski operator.

In the calculation of the average work dissipated during the generic $n_{c}$-th cycle (see Eq. 20), the contribution of the first two addends in Eq. 36 is null due to the periodicity of these terms. Thus,

$$
\bar{w}_{\text {diss }, n_{c}}=\bar{w}\left(0, t_{n_{c}}\right)-\bar{w}\left(0, t_{n_{c}-1}\right)=\sum_{n} \sum_{N, N^{\prime}}\left[\alpha_{n}\left(t_{n_{c}}, N, N^{\prime}\right)-\alpha_{n}\left(t_{n_{c}-1}, N, N^{\prime}\right)\right]
$$

By using Eq. 37, a few more steps yield

$$
\bar{w}_{\text {diss }, n_{c}}=-\omega^{2} \sum_{n} \sum_{N, N^{\prime}} \frac{N N^{\prime} v_{n}(N) v_{n}\left(N^{\prime}\right)}{\lambda_{n}+i \omega N^{\prime}}\left\{\tau \delta_{N^{\prime},-N}+\frac{e^{-\left(\lambda_{n}-i \omega N\right) t_{n_{c}-1}}}{\lambda_{n}-i \omega N}\left(e^{-\left(\lambda_{n}-i \omega N\right) \tau}-1\right)\right\}
$$

where $\delta$ stands for Kronecker's Delta function and $\tau=2 \pi / \omega$ is the perturbation period. The expression in the asymptotic limit, i.e. for $n_{c} \rightarrow \infty$ so that $t_{n_{c}-1} \rightarrow \infty$, can be obtained by considering that the second addend within the curly brackets behaves as follows (recall that the $\lambda_{n}$ are positive-valued for $n \geq 1$ ):

$$
\frac{e^{-\left(\lambda_{n}-i \omega N\right) t_{n_{c}-1}}}{\lambda_{n}-i \omega N}\left(e^{-\left(\lambda_{n}-i \omega N\right) \tau}-1\right)=\left\{\begin{array}{l}
0 \text { for } n=0, N \neq 0 \\
-\tau \text { for } n=0, N=0 \\
\rightarrow 0, \text { for } n \geq 1 \text {, as } n_{c} \rightarrow \infty
\end{array}\right.
$$


Thus, in Eq. 39 such a term is immaterial since it brings a null contribution for $n=0$ (and any $N$ ) or it vanishes in the asymptotic limit for $n \geq 1$. It follows that

$$
\lim _{n_{c} \rightarrow \infty} \bar{w}_{\text {diss }, n_{c}}=\bar{w}_{\text {diss }}^{\infty} \stackrel{(i)}{=} 2 \pi \omega \sum_{n} \sum_{N} \frac{N^{2}\left|v_{n}(N)\right|^{2}}{\lambda_{n}-i \omega N} \stackrel{(i i)}{=} 2 \pi \omega \sum_{n} \sum_{N=-\infty}^{+\infty} \frac{N^{2} \lambda_{n}\left|v_{n}(N)\right|^{2}}{\lambda_{n}^{2}+N^{2} \omega^{2}}
$$

where $v_{n}(-N)=v_{n}(N)^{*}$ has been recalled in step (i); step (ii) has been carried out by writing explicitly the real and the imaginary parts of the summation and recognizing that the imaginary part vanishes. The expression Eq. 21 readily follows by considering that the contribution for $n=0$ is null (since $\lambda_{0}=0$ ), then that the term $N=0$ is null while the symmetric terms for $\pm N$ are equal, and by employing the definitions of the frequencies $\omega_{N}$ and of the factors $Q(n, N)$.

\section{Compact form of $\bar{w}_{\text {diss }}^{\infty}$ and of its approximations at low and high frequencies}

Notably, one can re-compact Eqs. 21-23 in terms of operations that involve timecorrelation functions, at the unperturbed equilibrium, of the energy perturbation and of its rate of evolution. The results are summarized here below while the derivation is given in Appendix A.

Let us denote with $\delta V_{1}(\mathbf{x}, t)$ the deviation of the energy perturbation at time $t$ from its average value at the unperturbed equilibrium:

$$
\delta V_{1}(\mathbf{x}, t)=V_{1}(\mathbf{x}, t)-\left\langle V_{1}(\mathbf{x}, t)\right\rangle_{0}
$$

Then we introduce the rate of evolution of the energy perturbation,

$$
V^{(1)}(\mathbf{x}, t)=\frac{\partial V_{1}(\mathbf{x}, t)}{\partial t}
$$

and, as above, its deviation from the equilibrium average:

$$
\delta V^{(1)}(\mathbf{x}, t)=V^{(1)}(\mathbf{x}, t)-\left\langle V^{(1)}(\mathbf{x}, t)\right\rangle_{0}
$$

Finally, let $G_{\delta V_{1}\left(t^{\prime}\right), \delta V_{1}\left(t^{\prime \prime}\right)}(t)$ be the time-correlation function between $\delta V_{1}\left(\mathbf{x}, t^{\prime}\right)\left(t^{\prime}\right.$ as fixed parameter) and $\delta V_{1}\left(\mathbf{x}, t^{\prime \prime}\right)$ ( $t^{\prime \prime}$ as fixed parameter) at the unperturbed equilibrium.[29] Similarly, $G_{\delta V^{(1)}\left(t^{\prime}\right), \delta V^{(1)}\left(t^{\prime \prime}\right)}(t)$ is the time-correlation function between $\delta V^{(1)}\left(\mathbf{x}, t^{\prime}\right)$ ( $t^{\prime}$ as fixed parameter) and $\delta V^{(1)}\left(\mathbf{x}, t^{\prime \prime}\right)\left(t^{\prime \prime}\right.$ as fixed parameter) at the unperturbed equilibrium. In Appendix A we show that Eq. 21 can be converted in the following form:

$$
\begin{aligned}
\bar{w}_{\mathrm{diss}}^{\infty}(\omega) & \simeq \int_{0}^{\tau} d t^{\prime} \int_{0}^{t^{\prime}} d t^{\prime \prime} G_{\delta V^{(1)}\left(t^{\prime}\right), \delta V^{(1)}\left(t^{\prime \prime}\right)}\left(t^{\prime}-t^{\prime \prime}\right) \\
& +\sum_{m \geq 1} \int_{0}^{\tau} d t^{\prime} \int_{0}^{\tau} d t^{\prime \prime} G_{\delta V^{(1)}\left(t^{\prime}\right), \delta V^{(1)}\left(t^{\prime \prime}\right)}\left(m \tau-\left(t^{\prime}-t^{\prime \prime}\right)\right)
\end{aligned}
$$


We also demonstrate that the coefficients $c_{1}$ and $c_{2}$ in Eqs. 22 can be expressed as

$$
c_{1}=\frac{1}{2 \pi} \int_{0}^{\infty} d t \Phi_{1}(t) \quad, \quad c_{2}=\left.2 \pi \frac{d \Phi_{2}(t)}{d t}\right|_{t=0}
$$

where $\Phi_{1}(t)$ and $\Phi_{2}(t)$ are the following functions:

$$
\begin{aligned}
& \Phi_{1}(t)=\tau \int_{0}^{\tau} d t^{\prime} G_{\delta V^{(1)}\left(t^{\prime}\right), \delta V^{(1)}\left(t^{\prime}\right)}(t) \\
& \Phi_{2}(t)=\frac{1}{\tau} \int_{0}^{\tau} d t^{\prime}\left\{-G_{\delta V_{1}\left(t^{\prime}\right), \delta V_{1}\left(t^{\prime}\right)}(t)+\frac{1}{\tau} \int_{0}^{\tau} d t^{\prime \prime} G_{\delta V_{1}\left(t^{\prime}\right), \delta V_{1}\left(t^{\prime \prime}\right)}(t)\right\}
\end{aligned}
$$

Note that, although $\tau$ enters the expressions of $\Phi_{1}(t)$ and $\Phi_{2}(t)$, both functions are frequencyindependent. Finally, the upper bound in Eq. 23 takes the form

$$
\max _{\omega}\left\{\bar{w}_{\text {diss }}^{\infty}(\omega)\right\}<\sqrt{d \Phi_{2}(t) /\left.d t\right|_{t=0} \int_{0}^{\infty} d t \Phi_{1}(t)}
$$

Although the expressions given above may seem appealing per se, only their "disclosed" forms in Eqs. 21-23 are useful in uncovering the features of the system's response to the perturbation. Moreover, examining the derivation in Appendix A, the reader may realize that there are several alternatives to re-compact Eqs. 21-23 in terms of operations on suitable time-correlation functions. Also, by means of integrations by parts and substitutions one could further elaborate the expressions presented above and achieve different, but equivalent, relations (to some extent, it is a matter of taste where to stop the algebraic elaboration). On the other hand, an advantage of these compact forms is that the time-correlation functions involved could be directly computed, depending on the complexity of the system, from the generation of a sufficiently long trajectory at equilibrium (for example, for nanoscopic systems one could perform molecular dynamics or Langevin simulations).[29]

We would like to stress that Eq. 45 or alternative but equivalent forms could establish the connection with the framework of the non-equilibrium thermodynamics of periodically driven systems recently presented in ref. [10]. In the linear-response regime between "fluxes" and "affinities" suitably identified, [30] the authors derive the Onsager-like kinetic coefficients which enter the rate of average entropy production. By focusing on the case of fixed temperature (the theory in ref. [10] comprises also the possibility of temperature variations), the "affinity" associated to the work-flux is related to the magnitude of the energy perturbation. Although a detailed comparison has not been carried out here, both the average energy dissipation obtainable from integration of the average entropy production rate in ref. [10] (which is quadratic with respect to the affinity) and our Eq. 45 (where timecorrelation functions of the energy perturbation are involved), clearly pertain to the same order of approximation. [31] However, some discrepancies might be detected since the two approaches are different, and the linear approximation with respect to the energy perturbation is applied at different stages. 


\section{MODEL CALCULATIONS}

For a simple uni-dimensional system where the set $\mathbf{x}$ reduces to a single stochastic variable $x$, we compare the exact value of $\bar{w}_{\text {diss }}^{\infty}$ obtained by the numerical solution of the nonstationary Fokker-Planck-Smoluchowski equation, with the analytic approximation in Eq. 21. For completeness, some statistical properties of the energy dissipated per cycle in the asymptotic limit are discussed in Appendix B.

In what follows, all quantities are dimensionless, that is, they are meant to be expressed in some units of measure which are immaterial in this study. We recall that all energy quantities are expressed in $k_{B} T$ units.

\section{A. Model system and applied perturbations}

The model system considered here is a generalized "particle on a ring" whose unperturbed energy function is

$$
V_{0}(x)=\alpha_{1} \cos (x)+\alpha_{2} \cos (2 x)
$$

where $\alpha_{1}=1$ and $\alpha_{2}=3$ in the calculations. For simplicity's sake, we take a constant diffusion coefficient $D(x)=D$; in the calculations $D=1$.

Two kinds of energy perturbation are considered, both built by employing the single periodically evolving parameter

$$
\epsilon(t)=\Delta \sin \left(\omega_{\text {ext }} t\right)
$$

where $\Delta=4$ in the calculations. The two cases are

$$
\begin{aligned}
& \text { Case } 1: V_{1}(x, t)=\epsilon(t) \cos (x) \\
& \text { Case } 2: V_{1}(x, t)=\epsilon(t) \cos (x+\phi \epsilon(t) / \Delta)
\end{aligned}
$$

where $\phi=0.7$ in the calculations. With these kinds of perturbations we have that $\omega \equiv \omega_{\text {ext }}$ in both cases (see the note ref. [21]). In Case 1, a single Fourier component $N=1$ is present. In Case 2, the non-linear dependence on $\epsilon(t)$ implies that an infinite number of higher harmonics $\omega_{N}=N \omega$ is present.

In Case 1 the perturbation modulates the height of the central energy barrier but the symmetry of the potential around $x=\pi$ is preserved (although the locations of the minima change); in Case 2 the perturbation produces an asymmetry of the potential profile. Figure 2 displays the profile of $V_{0}(x)$ and the time evolution of $V(x, t)=V_{0}(x)+V_{1}(x, t)$, over one cycle, for both Cases.

\section{FIGURE 2}


Equilibrium fluctuations in a bi-stable potential is an extensively studied process in several regimes of motion; see for example ref. [23] for dynamics in the overdamped regime. The physical intuition leads us to imagine two kinds of processes: the slow thermally activated jump from one potential well to the other via crossing of the energy barrier and faster fluctuations inside the wells. These processes are related to the intrinsic "relaxation modes" introduced in Section II A through Eq. 12. Namely, it is well known that the smallest non-null eigenvalue of the Smoluchowski operator, $\lambda_{1}$, is separated by the higher ones. As the energy barrier increases, the separation increases $\left(\lambda_{1} \ll \lambda_{n \geq 2}\right)$ and $\lambda_{1}$ can be related to the kinetic rate of well-to-well transition, $k_{\text {cross }} \simeq \lambda_{1} / 2$, while the eigenfunction $\phi_{1}(x)$ corresponds to the relaxation mode associated to such a jump process. [23] The higher eigenvalues and the corresponding eigenfunctions are then associated to rates and modes of intra-well fluctuations. In the specific case, $\lambda_{1}=0.0243$ and $\lambda_{2}=7.640$.

\section{B. Simulation of stochastic trajectories}

The simulation of stochastic trajectories is performed here by implementing the Langevin equation in the overdamped regime of motion and by employing a white noise of Gaussian type.[32] Namely, a single propagation step is $x(t+\Delta t)=x(t)+D f(x, t) \Delta t+\sqrt{2 D \Delta t} \eta_{t}$ with $f(x, t)=-\partial V(x, t) / \partial x$ and where $\eta_{t}$ is a random number drawn from the Gaussian distribution with zero mean and unit variance. In all cases, the time-step $\Delta t$ was set equal to $10^{-4}$ (see remarks in the next section).

Some stochastic trajectories for the system at equilibrium and under perturbation are shown in Figure 3. All the trajectories displayed start from $x(0)=\operatorname{acos}\left(-\alpha_{1} / 4 \alpha_{2}\right)=1.654$ corresponding to the minimum of the left potential well of $V_{0}(x)$. The periodic boundary conditions at $x=0$ and $x=2 \pi$ are removed here to better show the excursions. In panel (a) the trajectories develop on the unperturbed potential landscape for a duration of $t_{\text {tot }}=50$. In panels (b) and (c) the trajectories develop, respectively, under the perturbations of Case 1 and Case 2 for ten cycles with $\tau=5$ (the total duration is still $t_{\text {tot }}=50$ ). As expected, the trajectories feature a small-steps motion mainly localized inside the potential wells, while well-to-well jumps occur on the longer timescale. Qualitatively it appears that, for both kinds of perturbation with that chosen period, the frequency of jumps is higher than that observed for the unperturbed system.

FIGURE 3 


\section{Numerical computation of the average dissipation per cycle}

The exact value of $\bar{w}_{\text {diss }, n_{c}}$ is computed by solving numerically the Fokker-PlanckSmoluchowski equation with initial condition $p(x, 0)=p_{e q, 0}(x)$. Periodic boundary conditions on the probability-density flux are applied at $x=0$ and $x=2 \pi$ in order to account for the circularity of the system. The solution is obtained by converting Eq. 13 into a discretized matricial format by employing a finite-differences scheme with uniform partition of the domain in intervals of width $\Delta x$. The resulting format is

$$
\dot{\mathbf{p}}=-\mathbf{M}(t) \mathbf{p}
$$

where $\mathbf{p}(t)$ is a column array of dimension equal to the number of mesh points $N_{p}$ and whose components are $p_{n}(t)=p\left(x_{n}, t\right)$, and where $\mathbf{M}(t)$ is the $N_{p} \times N_{p}$ time-dependent evolution matrix given in the note ref. [33] For the unperturbed system, the evolution matrix reduces to the constant $\mathbf{M}_{0}$ which corresponds to the operator $\hat{\Gamma}_{0}$. [33] The realsymmetric matrix $\tilde{\mathbf{M}}_{0}$ with elements $\left[\tilde{\mathbf{M}}_{0}\right]_{n n^{\prime}}=\left[\mathbf{M}_{0}\right]_{n n^{\prime}} e^{-\left[V_{0}\left(x_{n^{\prime}}\right)-V_{0}\left(x_{n}\right)\right] / 2}$ corresponds to $\tilde{\Gamma}_{0}$. The diagonalization of such a matrix yields the eigenvalues $\lambda_{n}\left(\tilde{\mathbf{M}}_{0}\right)$, and the related eigenvectors, which approximate eigenvalues/eigenfunctions of $\tilde{\Gamma}_{0}$.

The basic forward Euler propagator is applied to let evolve the array $\mathbf{p}(t)$ under perturbation. Namely, the advance of time $\Delta t$ is attained as $\mathbf{p}(t+\Delta t)=[\mathbf{I}-\Delta t \mathbf{M}(t)] \mathbf{p}(t)$ where I is the $N_{p} \times N_{p}$ identity matrix; a constant $\Delta t$ is adopted. At each step, the integration required in Eq. 20 is performed by employing the rectangles rule. Globally,

$$
\bar{w}_{\text {diss }, n_{c}} \simeq \Delta x \Delta t \sum_{i=1}^{N_{t}} \mathbf{p}\left(t_{i}\right)^{T} \mathbf{V}^{(1)}\left(t_{i}\right)
$$

where $\mathbf{V}^{(1)}\left(t_{i}\right)$ is the column array with elements

$$
\left[\mathbf{V}^{(1)}\left(t_{i}\right)\right]_{n}=\left.\frac{\partial V\left(x_{n}, t\right)}{\partial t}\right|_{t=t_{i}}
$$

and $N_{t}$ is the number of time-steps per cycle. Concerning the time-step of propagation, let $\Delta t_{0}$ be a target value; in order to account accurately for the fastest modes, it should be $\Delta t_{0} \leq 0.1 / \max _{n}\left\{\lambda_{n}\left(\tilde{\mathbf{M}}_{0}\right)\right\}$. Then we set $N_{t}=\operatorname{INT}\left(\tau / \Delta t_{0}\right)$, from which the actual timestep is obtained as $\Delta t=\tau / N_{t}$. The calculations have been performed with $N_{p}=100$ and $\Delta t \simeq \Delta t_{0}=10^{-4}$ (such a value meets the condition given above), making sure that a further increase of $N_{p}$ and decrease of $\Delta t$ do not significantly change the value $\bar{w}_{\text {diss, } n_{c}}$ for which the asymptotic limit is considered reached. The explored range of $\tau$ values is such that $\omega$ varies from $10^{-3}$ to $10^{3}$ for both Case 1 and Case 2 .

The asymptotic limit is considered achieved when the relative variation $\mid \bar{w}_{\text {diss }, n_{c}}-$

$\bar{w}_{\text {diss }, n_{c}-1} \mid / \bar{w}_{\text {diss }, n_{c}-1}$ falls below 0.001 . In nearly all cases (except for a very large $\tau$ ), the 
variation is much smaller than the first relative change from $\bar{w}_{\text {diss }, 1}$ to $\bar{w}_{\text {diss }, 2}$. Few cycles (generally less than 10) are required to reach convergence for both Case 1 and Case 2. We have also checked that, going further with the number of cycles, the possible change in the outcome is irrelevant. A further check of self-consistency and accuracy is provided by the full agreement between these outcomes and the values of $\bar{w}_{\text {diss }}^{\infty}$ computed as the average over a statistical ensemble of $w_{\text {diss }}^{\infty}$ values produced by the simulation of long stochastic trajectories (see the Appendix B).

With regard to the analytic approximation in Eq. 21, the computation of the $v_{n}(N)$ factors from Eq. 17 was performed via numerical integration.[34] It emerges that only the $v_{n}(1)$ components are not null for Case 1 . For Case 2 , all $v_{n}(N)$ with $N \geq 1$ do contribute although, in practice, the truncation at $N=10$ suffices to achieve numerical convergence.

\section{Average dissipation: analytical approximation vs. numerical solution}

In Figure 4 we show the trends of $\bar{w}_{\text {diss, } n_{c}}$ versus $n_{c}$ for the two kinds of perturbation. The calculations refer to $\tau=5$. For Case 1 , the asymptotic limit is essentially reached after only one cycle (note the small change from $\bar{w}_{\text {diss }, 1}$ to $\bar{w}_{\text {diss }, 2}$, in the order of $5 \%$ ). Moreover, the values show an increasing trend. For Case 2 the trend is instead decreasing, with a much lower rate of convergence and wider range of variation.

\section{FIGURE 4}

The main results are displayed in Figure 5 which shows, for Case 1 and Case 2, the dependence of $\bar{w}_{\text {diss }}^{\infty}$ on the frequency $\omega$. The circles correspond to the exact values from the numerical solution, while the solid line is the analytic approximation. The bars on the top axes display the first 30 eigenvalues of the matrix $\mathbf{M}_{0}$ in ascending order; these values approximate well the eigenvalues of the Smoluchowski operator (the largest eigenvalue, which is the most sensitive one to the matrix truncation, changes less than $5 \%$ passing from $N_{p}=100$ to $\left.N_{p}=200\right)$.

\section{FIGURE 5}

The exact and approximate solutions are indistinghishable for Case 1. For Case 2, the qualitative features (two maxima) of the profile are obtained, but the agreement is good only at high frequencies while the accuracy of the approximation degrades as $\omega$ decreases. 
Notably, the linear trends with slopes +1 and -1 , which appear for both Case 1 and Case 2 at low and high frequencies in the double logarithmic scale, agree with the limit behaviors in Eqs. 22. A characteristic pattern then emerges in the intermediate frequency range. In Case 1, where the perturbation mainly modulates the magnitude of the energy barrier but preserves the symmetry of $V_{0}(x)$, it appears that the average dissipation has a single maximum at a frequency in the range of the lower eigenvalues associated to fluctuations within the potential wells. In Case 2, for which the whole energy profile is distorted by the perturbation, the profile displays two maxima: again a maximum at a frequency of the order of the lower eigenvalues of the intra-well fluctuations, plus a further maximum at a frequency slightly smaller than $\lambda_{1}$, i.e., comparable with the well-to-well crossing rate.

These different profiles can be rationalized by following the qualitative arguments of Section III about the possibility of identifying a gap in the fluctuation rates if the external perturbation is "suitably tuned". A gap does indeed exist between the eigenvalue $\lambda_{1}$ and the much higher eigenvalues $\lambda_{n \geq 2}$. For Case 1, the expansion of $V_{1}(x, t)$ given in Eq. 14 with Eq. 16 involves only functions $g_{n}(x)$ of even symmetry with respect to the central point. The corresponding eigenfunctions $\phi_{n}(x)$ of $\tilde{\Gamma}_{0}$, which are related to (some of the) fast modes of fluctuation within the potential wells, belong to the set $n \geq 2$. In this case, $\bar{w}_{\text {diss }}^{\infty}$ features a single "band" to which only eigenvalues $\lambda_{n \geq 2}$ contribute. In Case 2 , also the function $g_{1}(x)$ with odd symmetry[23] contributes to the expansion of $V_{1}(x, t)$. In this situation, one of the two resolved bands corresponds to $\lambda_{1}$ and the other to the set of eigenvalues $\lambda_{n \geq 2}$.

Even if the overall behavior of the average dissipation is captured in both cases, the marked overestimation of $\bar{w}_{\text {diss }}^{\infty}$ which is found in the low-frequency range for Case 2 (up to a factor $\sim 5$ ) warns us about the possible quantitative failure of Eq. 21 when the system features activated process and the frequency of the perturbation is of the order of, or lower than, the corresponding kinetic rates.

\section{CONCLUDING REMARKS AND PERSPECTIVES}

This work has addressed the problem of finding an analytic approximation of the amount of energy that is dissipated per cycle when a system fluctuates (in the overdamped regime of motion) while it undergoes a cyclic transformation driven by an external perturbation which modulates the configurational energy. The main result is constituted by Eq. 21 which quantifies the interrelations depicted in Figure 1b; namely, it shows how the average dissipation per cycle, attained after many cycles, $\bar{w}_{\text {diss }}^{\infty}(\omega)$, is related to the relaxation rates of fluctuation at equilibrium, to the frequency $\omega$ of the modulation, and to the features of the energy perturbation. The equation is an approximation which is accurate for small enough energy perturbations, since it relies on the applicability of a first-order perturba- 
tive treatment with respect to the strength of the perturbation. A compact expression of $\bar{w}_{\text {diss }}^{\infty}(\omega)$, which involves characteristic time-correlation functions at equilibrium of the energy perturbation and of its rate of evolution, has also been obtained. We have pointed out that such a relation, or further elaborated forms, may establish a link with the expression of the average entropy production rate recently presented in ref. [10]. Moreover, we have provided an upper bound to the average dissipation per cycle. Although this result relies on the accuracy of the analytic approximation, to our knowledge the discovery of an upper bound is in itself an interesting fact since generally, with regard to dissipation, only a lower bound is obtained.

The numerical simulations performed on a toy model constituted by a "particle on a ring" which fluctuates in a bi-stable energy profile perturbed in two ways, have shown that the analytic approximation is accurate or, in any case, it is able to capture the essential traits of the true profile of $\bar{w}_{\text {diss }}^{\infty}(\omega)$. We stress again that Eq. 21 has been derived by applying the lowest order approximation to the non-equilibrium distribution $p(\mathbf{x}, t)$. Moving to the next order of approximation (re-starting from Eq. 26 in Section III B), some heavier algebra would lead to a more accurate expression for $\bar{w}_{\text {diss }}^{\infty}(\omega)$, but the simplicity of Eq. 21 would be lost. As a supplementary analysis of the model cases, we inspected how some general features of the distribution function $p\left(w_{\text {diss }}^{\infty}\right)$ depend on the frequency of the perturbation (results presented in Appendix B).

We are convinced that the current study may point to several practical and speculative pathways, mainly regarding the employment of the approximation Eq. 21; two of them are indicated here below.

From a practical point of view one could explore the possibility of devising a sort of "spectroscopic calorimetry" in which the average amount of dissipated energy is evaluated by measuring the heat exchanged between the thermal bath and a macroscopic ensemble of independent systems (i.e., non-interacting identical replicas) simultaneously subjected to the perturbation. This clearly excludes direct single-molecule mechanical manipulations. Rather, one could think, hypothetically, about situations in which the energetics of each replica can be modulated via interaction with some controlled parameter/property of a physical support to which the replicas are tethered; for example, it might be the case of nanosystems deposited on metallic surfaces whose electrostatic potential is externally varied in a periodic way. Another situation could be that of an ensemble of mesoscopic systems exposed to external periodic fields (an example is discussed below). In these cases, there is only a scale factor between the measurable exchanged heat per cycle and $\bar{w}_{\text {diss }}^{\infty}(\omega)$. The frequency profile of $\bar{w}_{\text {diss }}^{\infty}(\omega)$ could feature characteristic bands to be associated with compact sets of fluctuation rates, as discussed in Section III. With this kind of experiment one could probe the internal energetics/dynamics of the unperturbed system, and, specifically, achieve 
basic information about the presence of timescale separations.

It must be stressed here that the entire elaboration refers to general "systems" with no specification given about their nature, except for the fact that they are classical and that thermal fluctuations play a role. Indeed they can range from molecular/supra-molecular aggregates to mesoscopic objects. An example of the latter category is that of phospholipidic vesicles suspended in viscous solvents. Thermal fluctuations appear in terms of stochastic rearrangements of the shape (see for example the basic theory of Milner and Safran for quasi-spherical fluctuations [35]). Moreover, the conformational energy of the whole vesicle depends, in addition to the intrinsic elastic parameters [36], also on exposure to electric $[37,38]$ and magnetic [39] fields; this brings a contribution to the free energy per unit area of the vesicle. For example, alternated electric fields are known to affect the morphology and the dynamic behavior of giant vesicles and cells (see, for example, the review ref. [40] and references therein). A spectroscopic-like experiment in which the average energy dissipation is determined at different field frequencies, could shed light on the intrinsic modes/rates of fluctuation of the unperturbed vesicle.[41]

A further and more speculative line of research could be that of employing the explicit form of $\bar{w}_{\text {diss }}^{\infty}(\omega)$ to build an objective function for the "optimization" of the unperturbed system in the space of its parameters which determine energetics (configurational energy $V_{0}(\mathbf{x})$ ) and friction (diffusion matrix $\mathbf{D}(\mathbf{x})$ ). In fact, these are the only physical ingredients which affect the eigenfunctions and eigenvalues of the Smoluchowski operator for the unperturbed system. The question is: given an external agent able to interact with some property of the system in a periodic way, can we think about a strategy to optimize the system such as to produce a dissipation profile $\bar{w}_{\text {diss }}^{\infty}(\omega)$ with desired features? The perspective can also be turned around: given the system, can we tune the kind of perturbation in such a way that the dissipation profile shows the desired properties? Clearly, this kind of study can be undertaken only once physically-based criteria are provided giving a meaning to the words "optimization" and "tuning" used above. For example, Schmiedl and Seifert[42] opened the door to the idea of designing "optimal protocols", of given duration, targeted to minimize the average energy dissipation in driven non-equilibrium transformations; [43] if the objective were to minimize $\bar{w}_{\text {diss }}^{\infty}(\omega)$, a suitably "tuned" cyclic energy perturbation would correspond to an optimal protocol whose features might be, in all generality, $\omega$-dependent.

\section{Acknowledgements}

The authors would like to thank Linda Inverso for her assistance in preparing the English 
text for publication.

\section{Appendix A. Derivation of Eqs. 45 and 46}

In the derivations we will make use of the following forms of the time-correlation functions $G_{\delta V_{1}\left(t^{\prime}\right), \delta V_{1}\left(t^{\prime \prime}\right)}(t)$ and $G_{\delta V^{(1)}\left(t^{\prime}\right), \delta V^{(1)}\left(t^{\prime \prime}\right)}(t)$ in which the symmetrized Smoluchowski operator is involved:[29]

$$
G_{\delta V_{1}\left(t^{\prime}\right), \delta V_{1}\left(t^{\prime \prime}\right)}(t)=\int d \mathbf{x} \delta V_{1}\left(\mathbf{x}, t^{\prime}\right) p_{e q, 0}(\mathbf{x})^{1 / 2} e^{-t \tilde{\Gamma}_{0}} p_{e q, 0}(\mathbf{x})^{1 / 2} \delta V_{1}\left(\mathbf{x}, t^{\prime \prime}\right)
$$

and

$$
G_{\delta V^{(1)}\left(t^{\prime}\right), \delta V^{(1)}\left(t^{\prime \prime}\right)}(t)=\int d \mathbf{x} \delta V^{(1)}\left(\mathbf{x}, t^{\prime}\right) p_{e q, 0}(\mathbf{x})^{1 / 2} e^{-t \tilde{\Gamma}_{0}} p_{e q, 0}(\mathbf{x})^{1 / 2} \delta V^{(1)}\left(\mathbf{x}, t^{\prime \prime}\right)
$$

In these expressions, the times $t^{\prime}$ and $t^{\prime \prime}$ are fixed parameters.

Derivation of $\boldsymbol{E q}$. 45. Let us start by considering that Eq. 21 can be converted in the following compact form (see the proof in note [44]):

$$
\bar{w}_{\mathrm{diss}}^{\infty}=2 \pi \omega \frac{1}{\tau^{2}} \int_{0}^{\tau} d t^{\prime} \int_{0}^{\tau} d t^{\prime \prime} \int_{0}^{\infty} d t G_{\delta V_{1}\left(t^{\prime}\right), \delta V_{1}\left(t^{\prime \prime}\right)}(t) \sigma\left(t^{\prime}, t^{\prime \prime}, t\right)
$$

where $G_{\delta V_{1}\left(t^{\prime}\right), \delta V_{1}\left(t^{\prime \prime}\right)}(t)$ is the time-correlation function given in Eq. A1, and where

$$
\sigma\left(t^{\prime}, t^{\prime \prime}, t\right)=\sum_{N=-\infty}^{+\infty} N^{2} e^{-i \omega N\left(t^{\prime}-t^{\prime \prime}+t\right)}
$$

Let us now express the function $\sigma\left(t^{\prime}, t^{\prime \prime}, t\right)$ in the following equivalent form:

$$
\sigma\left(t^{\prime}, t^{\prime \prime}, t\right) \equiv \frac{1}{\omega^{2}} \frac{\partial^{2}}{\partial t^{\prime} \partial t^{\prime \prime}}\left\{\sum_{N=-\infty}^{+\infty} e^{-i \omega N\left(t^{\prime}-t^{\prime \prime}+t\right)}\right\}
$$

The summation into curly brackets corresponds to a train of Dirac's Delta Functions (which are denoted hereafter by $\left.\delta_{D}\right)$ :

$$
\sum_{N=-\infty}^{+\infty} e^{-i \omega N t_{x}} \equiv \frac{2 \pi}{\omega} \sum_{m=-\infty}^{+\infty} \delta_{D}\left(t_{x}-m \tau\right)
$$


with $t_{x}=t^{\prime}-t^{\prime \prime}+t$ in the present case. By inserting Eq. A5 with Eq. A6 into Eq. A3, the multiplicative factors cancel and

$$
\bar{w}_{\mathrm{diss}}^{\infty}=\sum_{m=-\infty}^{+\infty} \int_{0}^{\infty} d t \int_{0}^{\tau} d t^{\prime} \int_{0}^{\tau} d t^{\prime \prime} G_{\delta V_{1}\left(t^{\prime}\right), \delta V_{1}\left(t^{\prime \prime}\right)}(t) \frac{\partial^{2} \delta_{D}\left(t^{\prime}-t^{\prime \prime}+t-m \tau\right)}{\partial t^{\prime} \partial t^{\prime \prime}}
$$

The integral over $t^{\prime \prime}$ is now solved by parts:

$$
\begin{aligned}
& \int_{0}^{\tau} d t^{\prime \prime} G_{\delta V_{1}\left(t^{\prime}\right), \delta V_{1}\left(t^{\prime \prime}\right)}(t) \frac{\partial^{2} \delta_{D}\left(t^{\prime}-t^{\prime \prime}+t-m \tau\right)}{\partial t^{\prime} \partial t^{\prime \prime}}= \\
& =\left.G_{\delta V_{1}\left(t^{\prime}\right), \delta V_{1}\left(t^{\prime \prime}\right)}(t) \frac{\partial \delta_{D}\left(t^{\prime}-t^{\prime \prime}+t-m \tau\right)}{\partial t^{\prime}}\right|_{t^{\prime \prime}=0} ^{t^{\prime \prime}=\tau} \\
& -\int_{0}^{\tau} d t^{\prime \prime} \frac{\partial G_{\delta V_{1}\left(t^{\prime}\right), \delta V_{1}\left(t^{\prime \prime}\right)}(t)}{\partial t^{\prime \prime}} \frac{\partial \delta_{D}\left(t^{\prime}-t^{\prime \prime}+t-m \tau\right)}{\partial t^{\prime}}
\end{aligned}
$$

The first addend on the right-hand side is null since $V_{1}\left(\mathbf{x}, t^{\prime \prime}\right)$ is zero at $t^{\prime \prime}=0$ and $t^{\prime \prime}=\tau$, hence also the correlation function $G_{\delta V_{1}\left(t^{\prime}\right), \delta V_{1}\left(t^{\prime \prime}\right)}(t)$ is null at these times. Thus,

$$
\bar{w}_{\mathrm{diss}}^{\infty}=-\sum_{m=-\infty}^{+\infty} \int_{0}^{\infty} d t \int_{0}^{\tau} d t^{\prime} \int_{0}^{\tau} d t^{\prime \prime} \frac{\partial G_{\delta V_{1}\left(t^{\prime}\right), \delta V_{1}\left(t^{\prime \prime}\right)}(t)}{\partial t^{\prime \prime}} \frac{\partial \delta_{D}\left(t^{\prime}-t^{\prime \prime}+t-m \tau\right)}{\partial t^{\prime}}
$$

The integrations over $t^{\prime}$ and $t^{\prime \prime}$ are exchangeable, so that the integral over $t^{\prime}$ can be carried out first and solved by parts:

$$
\begin{aligned}
& \int_{0}^{\tau} d t^{\prime} \frac{\partial G_{\delta V_{1}\left(t^{\prime}\right), \delta V_{1}\left(t^{\prime \prime}\right)}(t)}{\partial t^{\prime \prime}} \frac{\partial \delta_{D}\left(t^{\prime}-t^{\prime \prime}+t-m \tau\right)}{\partial t^{\prime}}= \\
& =\left.\frac{\partial G_{\delta V_{1}\left(t^{\prime}\right), \delta V_{1}\left(t^{\prime \prime}\right)}(t)}{\partial t^{\prime \prime}} \delta_{D}\left(t^{\prime}-t^{\prime \prime}+t-m \tau\right)\right|_{t^{\prime}=0} ^{t^{\prime}=\tau} \\
& -\int_{0}^{\tau} d t^{\prime} \frac{\partial^{2} G_{\delta V_{1}\left(t^{\prime}\right), \delta V_{1}\left(t^{\prime \prime}\right)}(t)}{\partial t^{\prime} \partial t^{\prime \prime}} \delta_{D}\left(t^{\prime}-t^{\prime \prime}+t-m \tau\right)
\end{aligned}
$$

As above, the first addend is null since $V_{1}\left(\mathbf{x}, t^{\prime}\right)$ is zero at $t^{\prime}=0$ and $t^{\prime}=\tau$, hence also the correlation function $G_{\delta V_{1}\left(t^{\prime}\right), \delta V_{1}\left(t^{\prime \prime}\right)}(t)$, and its derivative with respect to $t^{\prime \prime}$ as well, are null at these times. Thus,

$$
\bar{w}_{\mathrm{diss}}^{\infty}=\sum_{m=-\infty}^{+\infty} \int_{0}^{\infty} d t \int_{0}^{\tau} d t^{\prime} \int_{0}^{\tau} d t^{\prime \prime} \frac{\partial^{2} G_{\delta V_{1}\left(t^{\prime}\right), \delta V_{1}\left(t^{\prime \prime}\right)}(t)}{\partial t^{\prime} \partial t^{\prime \prime}} \delta_{D}\left(t^{\prime}-t^{\prime \prime}+t-m \tau\right)
$$

The integral over $t$ in Eq. A11 is solved by exploiting the property of the Delta function. Namely, such an integral reduces to a sum of terms, each of them given by the integrand evaluated at times $t=m \tau-\left(t^{\prime}-t^{\prime \prime}\right)$. The $t \geq 0$ requirement, and the consideration that $-\tau \leq t^{\prime}-t^{\prime \prime} \leq+\tau$, put limitations on the admissible values of the integers $m$. Namely, values $m \leq-2$ are not admitted; the value $m=-1$ gives a null contribution (since only 
the situation $t^{\prime}=0, t^{\prime \prime}=\tau, t=0$ would be selected and the integral is zero in this case); $m=0$ requires that $t^{\prime} \leq t^{\prime \prime}$; values $m \geq 1$ do no require any constraint. The contribution for $m=0$ is

$$
\begin{aligned}
m=0 \leftrightarrow & \left.(i) \int_{0}^{\tau} d t^{\prime \prime} \int_{0}^{t^{\prime \prime}} d t^{\prime} \frac{\partial^{2} G_{\delta V_{1}\left(t^{\prime}\right), \delta V_{1}\left(t^{\prime \prime}\right)}(t)}{\partial t^{\prime} \partial t^{\prime \prime}}\right|_{t=-\left(t^{\prime}-t^{\prime \prime}\right)} \\
& \left.\stackrel{(i i)}{\equiv} \int_{0}^{\tau} d t^{\prime} \int_{0}^{t^{\prime}} d t^{\prime \prime} \frac{\partial^{2} G_{\delta V_{1}\left(t^{\prime \prime}\right), \delta V_{1}\left(t^{\prime}\right)}(t)}{\partial t^{\prime} \partial t^{\prime \prime}}\right|_{t=t^{\prime}-t^{\prime \prime}} \\
& \left.\stackrel{(i i i)}{=} \int_{0}^{\tau} d t^{\prime} \int_{0}^{t^{\prime}} d t^{\prime \prime} \frac{\partial^{2} G_{\delta V_{1}\left(t^{\prime}\right), \delta V_{1}\left(t^{\prime \prime}\right)}(t)}{\partial t^{\prime} \partial t^{\prime \prime}}\right|_{t=t^{\prime}-t^{\prime \prime}}
\end{aligned}
$$

where in the form $(i)$ the integrations over $t^{\prime}$ and $t^{\prime \prime}$ have been exchanged and the constraint $t^{\prime} \leq t^{\prime \prime}$ has been applied; in the form (ii) the labels $t^{\prime}$ and $t^{\prime \prime}$ have been exchanged; in the form (iii) we have exploited the fact a the time-correlation is invariant with respect to the switch of the order of the correlated functions if they are real-valued and the dynamics is a diffusive process (these conditions are met here). As a whole, the following result is obtained:

$$
\begin{aligned}
\bar{w}_{\mathrm{diss}}^{\infty} & =\left.\int_{0}^{\tau} d t^{\prime} \int_{0}^{t^{\prime}} d t^{\prime \prime} \frac{\partial^{2} G_{\delta V_{1}\left(t^{\prime}\right), \delta V_{1}\left(t^{\prime \prime}\right)}(t)}{\partial t^{\prime} \partial t^{\prime \prime}}\right|_{t=t^{\prime}-t^{\prime \prime}} \\
& +\left.\sum_{m \geq 1} \int_{0}^{\tau} d t^{\prime} \int_{0}^{\tau} d t^{\prime \prime} \frac{\partial^{2} G_{\delta V_{1}\left(t^{\prime}\right), \delta V_{1}\left(t^{\prime \prime}\right)}(t)}{\partial t^{\prime} \partial t^{\prime \prime}}\right|_{t=m \tau-\left(t^{\prime}-t^{\prime \prime}\right)}
\end{aligned}
$$

The final step consists in exploiting the following identity:

$$
\frac{\partial^{2} G_{\delta V_{1}\left(\mathbf{X}, t^{\prime}\right), \delta V_{1}\left(\mathbf{X}, t^{\prime \prime}\right)}(t)}{\partial t^{\prime} \partial t^{\prime \prime}} \equiv G_{\partial \delta V_{1}\left(\mathbf{X}, t^{\prime}\right) / \partial t^{\prime}, \partial \delta V_{1}\left(\mathbf{X}, t^{\prime \prime}\right) / \partial t^{\prime \prime}}(t) \equiv G_{V^{(1)}\left(t^{\prime}\right), V^{(1)}\left(t^{\prime \prime}\right)}(t)
$$

where Eqs. 42 and 44 have been recalled. Thus, Eq. 45 follows.

Derivation of the factors $c_{1}$ and $c_{2}$ in $\boldsymbol{E q}$. 46. Let us consider the following expansion (see the note [44]):

$$
\delta V_{1}(\mathbf{x}, t)=\sum_{N=-\infty}^{+\infty} \sum_{n \geq 1} v_{n}(N) g_{n}(\mathbf{x}) e^{i N \omega t}
$$

where the functions $g_{n}(\mathbf{x})$ have been introduced in Section II A. The time derivative yields $\delta V^{(1)}(\mathbf{x}, t)=i \omega \sum_{N=-\infty}^{+\infty} \sum_{n \geq 1} N v_{n}(N) g_{n}(\mathbf{x}) e^{i N \omega t}$. In what follows we shall deal with the time-correlation function $G_{\delta V_{1}\left(t^{\prime}\right), \delta V_{1}\left(t^{\prime \prime}\right)}(t)$ expanded as (see the note [44])

$$
G_{\delta V_{1}\left(t^{\prime}\right), \delta V_{1}\left(t^{\prime \prime}\right)}(t)=\sum_{N_{1}, N_{2}=-\infty}^{+\infty} \sum_{n \geq 1} v_{n}\left(N_{1}\right)^{*} v_{n}\left(N_{2}\right) e^{i \omega\left(-N_{1} t^{\prime}+N_{2} t^{\prime \prime}\right)} e^{-\lambda_{n} t}
$$


and with its expression for $t^{\prime \prime}=t^{\prime}$,

$$
G_{\delta V_{1}\left(t^{\prime}\right), \delta V_{1}\left(t^{\prime}\right)}(t)=\sum_{N_{1}, N_{2}=-\infty}^{+\infty} \sum_{n \geq 1} v_{n}\left(N_{1}\right)^{*} v_{n}\left(N_{2}\right) e^{i \omega\left(-N_{1}+N_{2}\right) t^{\prime}} e^{-\lambda_{n} t}
$$

Moreover, we shall also deal with the time-correlation function $G_{\delta V^{(1)}\left(t^{\prime}\right), \delta V^{(1)}\left(t^{\prime}\right)}(t)$ which is expanded (using Eq. A15) as

$$
G_{\delta V^{(1)}\left(t^{\prime}\right), \delta V^{(1)}\left(t^{\prime}\right)}(t)=\omega^{2} \sum_{N_{1}, N_{2}=-\infty}^{+\infty} \sum_{n, n^{\prime} \geq 1} N_{1} N_{2} v_{n}\left(N_{1}\right)^{*} v_{n^{\prime}}\left(N_{2}\right) e^{i\left(-N_{1}+N_{2}\right) \omega t^{\prime}} G_{g_{n}, g_{n^{\prime}}}(t)
$$

where $G_{g_{n}, g_{n^{\prime}}}(t)$ is the time-correlation function between $g_{n}(\mathbf{x})$ and $g_{n^{\prime}}(\mathbf{x})$ at the unperturbed equilibrium. Since $G_{g_{n}, g_{n^{\prime}}}(t)=\delta_{n, n^{\prime}} e^{-\lambda_{n} t}$ (see the remark in note[44]), it follows

$$
G_{\delta V^{(1)}\left(t^{\prime}\right), \delta V^{(1)}\left(t^{\prime}\right)}(t)=\omega^{2} \sum_{N_{1}, N_{2}=-\infty}^{+\infty} \sum_{n \geq 1} N_{1} N_{2} v_{n}\left(N_{1}\right)^{*} v_{n}\left(N_{2}\right) e^{i\left(-N_{1}+N_{2}\right) \omega t^{\prime}} e^{-\lambda_{n} t}
$$

Let us now recall the function $\Phi_{1}(t)$ given in Eqs. 47. By inserting Eq. A19 into that definition of $\Phi_{1}(t)$, when integrating over $t^{\prime}$, the complex factor $e^{i \omega\left(-N_{1}+N_{2}\right) t^{\prime}}$ gives $\tau \delta_{N_{1}, N_{2}}$. Thus,

$$
\Phi_{1}(t)=\omega^{2} \tau^{2} \sum_{N=-\infty}^{+\infty} \sum_{n \geq 1}\left|N v_{n}(N)\right|^{2} e^{-\lambda_{n} t} \equiv 2(2 \pi)^{2} \sum_{N=1}^{+\infty} \sum_{n \geq 1}\left|N v_{n}(N)\right|^{2} e^{-\lambda_{n} t}
$$

The integration of $\Phi_{1}(t)$ over $t$ from 0 to $\infty$ then yields

$$
\int_{0}^{\infty} d t \Phi_{1}(t)=8 \pi^{2} \sum_{N=1}^{+\infty} \sum_{n \geq 1} \lambda_{n}^{-1}\left|N v_{n}(N)\right|^{2}
$$

The comparison of Eq. A21 with $c_{1}$ given in Eqs. 22 leads us to state that $c_{1}=$ $(2 \pi)^{-1} \int_{0}^{\infty} d t \Phi_{1}(t)$, which the expression in Eq. 46.

Let us now define the following functions:

$$
\chi_{a}(t):=\frac{1}{\tau} \int_{0}^{\tau} d t^{\prime} G_{\delta V_{1}\left(t^{\prime}\right), \delta V_{1}\left(t^{\prime}\right)}(t) \quad, \quad \chi_{b}(t):=\frac{1}{\tau^{2}} \int_{0}^{\tau} d t^{\prime} \int_{0}^{\tau} d t^{\prime \prime} G_{\delta V_{1}\left(t^{\prime}\right), \delta V_{1}\left(t^{\prime \prime}\right)}(t)
$$

By inserting Eq. A17 into $\chi_{a}(t)$, when integrating over $t^{\prime}$, the complex factor $e^{i \omega\left(-N_{1}+N_{2}\right) t^{\prime}}$ gives $\tau \delta_{N_{1}, N_{2}}$. Thus,

$$
\chi_{a}(t)=\frac{1}{\tau} \times \tau \sum_{N=-\infty}^{+\infty} \sum_{n \geq 1}\left|v_{n}(N)\right|^{2} e^{-\lambda_{n} t} \equiv \sum_{n \geq 1}\left|v_{n}(0)\right|^{2} e^{-\lambda_{n} t}+2 \sum_{N=1}^{+\infty} \sum_{n \geq 1}\left|v_{n}(N)\right|^{2} e^{-\lambda_{n} t}
$$

Let us now take the time derivative of $\chi_{a}(t)$ and evaluate it at $t=0$ :

$$
\left.\frac{d \chi_{a}(t)}{d t}\right|_{t=0}=-\sum_{n \geq 1} \lambda_{n}\left|v_{n}(0)\right|^{2}-2 \sum_{N=1}^{+\infty} \sum_{n \geq 1} \lambda_{n}\left|v_{n}(N)\right|^{2}
$$


By recalling the expression of $c_{2}$ given in Eqs. 22, it follows that

$$
c_{2}=-2 \pi\left[\left.\frac{d \chi_{a}(t)}{d t}\right|_{t=0}+\sum_{n \geq 1} \lambda_{n}\left|v_{n}(0)\right|^{2}\right]
$$

Now it can be shown [45] that

$$
\sum_{n \geq 1} \lambda_{n}\left|v_{n}(0)\right|^{2} \equiv-\left.\frac{d \chi_{b}(t)}{d t}\right|_{t=0}
$$

The combination of Eqs. A25 and A26 yields

$$
c_{2}=2 \pi\left[\left.\frac{d \chi_{b}(t)}{d t}\right|_{t=0}-\left.\frac{d \chi_{a}(t)}{d t}\right|_{t=0}\right]
$$

By introducing $\Phi_{2}(t)=\chi_{b}(t)-\chi_{a}(t)$, which corresponds to the definition of $\Phi_{2}(t)$ given in Eqs. 47, it follows the final result $c_{2}=\left.2 \pi \frac{d \Phi_{2}(t)}{d t}\right|_{t=0}$.

\section{Appendix B. Work distribution functions: simulations results and expectations in} the low average dissipation limit

Let $p\left(w_{\text {diss }}^{\infty}\right)$ be the distribution function of the work dissipated per cycle after a very large number of cycles has been performed and a "periodic steady state" has been reached (i.e., the non-equilibrium distribution on the $\mathbf{x}$ variables evolves coherently with the perturbation). In principle, work distribution functions in driven transformations starting from equilibrium can be obtained by applying the strategy devised by Imparato et al. in ref. [46]; similarly, one could achieve the distribution $p\left(w_{\text {diss }}^{\infty}\right)$ of interest here. However, except for simple cases, such a route is challenging since it makes it necessary to solve partial differential equations for joint probability distributions on both work and $\mathbf{x}$ variables. This goes beyond the scope of this work [47]. In what follows, we report only some empirical evidence from the simulations on the model cases treated here.

In Figures 6 and 7 we show, for Case 1 and Case 2 respectively, the profiles of $p\left(w_{\text {diss }}^{\infty}\right)$ for four values of the frequency $\omega$ in the range of 0.1 to 100 . The circles correspond to the distributions obtained as follows. An initial value $x(0)$ was randomly drawn from $p_{e q, 0}(x)$. Then, a long Langevin trajectory under perturbation at the given $\omega$ was generated up to perform 11000 cycles. The first $10^{3}$ cycles served to assure the achievement of the asymptotic limit, while the amounts of work performed in the subsequent $10^{4}$ cycles were used to build 
$p\left(w_{\text {diss }}^{\infty}\right)$ by histograms. [48] For consistency, we checked that the values of $\bar{w}_{\text {diss }}^{\infty}$ computed by averaging over the $10^{4}$ outcomes perfectly fit within the trend of the data displayed in Figure 4, which were obtained from the time-propagation route described in section IV C.

FIGURE 6

\section{FIGURE 7}

For Case 1, from low to high frequency all work distributions appear uni-modal, while for Case 2 the distributions are bi-modal. We mention that this kind of bi-modal profile, that is a low-work narrow peak and a broader band at higher work values, was also found in ref. [7] where the authors experimentally investigated the case of a colloidal particle trapped in a double-well potential and subjected to a periodic external force which breaks the even symmetry, as takes place in our Case 2. The similarity of findings suggests us that the main features of $p\left(w_{\text {diss }}^{\infty}\right)$ are due to the bi-stability and to its symmetry loss, regardless of the subtle details of $V_{0}(x)$ and of the perturbation.

The solid curves displayed in some of the panels of Figures 6 and 7 represent the Gaussian distributions $p\left(w_{\text {diss }}^{\infty}\right) \simeq\left(\sigma_{\infty} \sqrt{2 \pi}\right)^{-1} \exp \left\{-\left(w_{\text {diss }}^{\infty}-\bar{w}_{\text {diss }}^{\infty}\right)^{2} / 2 \sigma_{\infty}^{2}\right\}$ with variance $\sigma_{\infty}^{2}=2 \bar{w}_{\text {diss }}^{\infty}$ and $\bar{w}_{\text {diss }}^{\infty}$ computed with the analytic approximation Eq. 21. Gaussian distributions with such a parametrization are expected in the low-dissipation limit in which the "lag" between $p(\mathbf{x}, t)$ and the "underlying" $p_{e q, t}(\mathbf{x})$ is small. This condition is intuitively met as $\tau$ increases, i.e., as the energy modulation becomes slower.[49] On the other hand, our simulations show that there are also cases where $p\left(w_{\text {diss }}^{\infty}\right)$ is close to the Gaussian profile even in the opposite small- $\tau$ range. This is evident for Case 2 where the largest deviation is observed at the frequency $\omega=0.1$ close to $\lambda_{1}$, while the Gaussian approximation becomes acceptable as $\omega$ increases (profiles of $p\left(w_{\text {diss }}^{\infty}\right)$ in the opposite range $\omega \ll \lambda_{1}$ have not been inspected). In all cases, and regardless of the period of the perturbation, $\bar{w}_{\text {diss }}^{\infty}$ in $k_{B} T$ units proves to be a global indicator of applicability of the Gaussian approximation. In the present case of a bi-stable system with a single degree of freedom, it appears that the Gaussian approximation can be accurate when $\bar{w}_{\text {diss }}^{\infty}$ is below half $k_{B} T$.

Finally, we checked the fulfillment of the relation $p\left(w_{\text {diss }}^{\infty}\right) / p\left(-w_{\text {diss }}^{\infty}\right) \simeq e^{w_{\text {diss }}^{\infty}}$. This relation is a form of the so-called "steady state fluctuation theorem" (SSFT), which constitutes an adaptation of the analogous expression known as "transient fluctuation theorem" (TFT) (see for example ref. [4] and references therein). In the TFT, the amount of work is referred to transformations starting from the system at equilibrium, while in the SSFT one focuses 
on a system which has reached a steady state (under perturbation) and is monitored over a finite time $\tau$. While the TFT holds exactly, the SSFT is expected to be generally fulfilled only approximately and with an accuracy which depends on the duration $\tau$. [50] The results are shown in Figures 8 and 9 where $\ln \left[p\left(w_{\text {diss }}^{\infty}\right) / p\left(-w_{\text {diss }}^{\infty}\right)\right]$ is plotted versus $w_{\text {diss }}^{\infty}$ in order to check if a linear correlation with unitary slope is present. What emerges is that the relation is well fulfilled for $w_{\text {diss }}^{\infty}$ small enough in absolute value (roughly, less than 2 in $k_{B} T$ units); for larger values, no certain affirmations can be made since the limited statistics that is available leads to badly reproduce the "wings" of the distributions making the data appear scattered.

\section{FIGURE 8}

FIGURE 9

\section{References and notes}

[1] K. Sekimoto, 'Stochastic energetics', Lecture notes in Physics 799 (Springer, Berlin Heidelberg, 2010).

[2] E. M. Sevick, R. Prabhakar, S. R. Williams, and D. J. Searles, Annu. Rev. Phys. Chem. 59, 603 (2008).

[3] C. Jarzynski, Annu. Rev. Condens. Matter Phys. 2, 329 (2011).

[4] S. Ciliberto, R. Gomez-Solano, and A. Petrosyan, Annu. Rev. Condens. Matter Phys. 4, 235 (2013).

[5] C. Jarzynski, The European Physical Journal B 64, 331 (2008).

[6] S. Saikia, R. Roy, and A. M. Jayannavar, Physics Letters A 369, 367 (2007).

[7] P. Jop, A. Petrosyan, and S. Ciliberto, EPL p. 50005 (2008).

[8] T. Harada and S. i. Sasa, Phys. Rev. Lett. 95, 130602 (2005).

[9] T. Harada and S. i. Sasa, Phys. Rev. E 73, 026131 (2006).

[10] K. Brandner, K. Saito, and U. Seifert, Phys. Rev. X 5, 031019 (2015).

[11] K. Proesmans and C. V. den Broek, Phys. Rev. Lett 115, 090601 (2015). 
[12] R. Zwanzig, Nonequilibrium Statistical Mechanics (Oxford University Press, New York, 2001).

[13] The configurational energies $V_{0}(\mathbf{x})$ and $V(\mathbf{x}, t)$ arise by performing a Boltzmann averaging over all fast-relaxing variables (the momenta are included in such a set), hence they are, strictly speaking, mean-field potentials (i.e., Helmholtz free energies of the configurational microstates).

[14] L. Peliti, J. Stat. Mech.: Theory and Experiment p. P05002 (2008).

[15] G. E. Crooks, J. Phys. Chem. 130, 107101 (2009).

[16] H. B. Callen, Thermodynamics and an introduction to Thermostatistics (Wiley, 1985).

[17] C. Jarzynski, Phys. Rev. Lett. 78, 2690 (1997).

[18] C. Jarzynski, Phys. Rev. E 56, 5018 (1997).

[19] S. Vaikuntanathan and C. Jarzynski, EPL 87, 60005 (2009).

[20] Namely, $\mathcal{D}\left[p(\mathbf{x}, t) \| p_{\text {eq,t }}(\mathbf{x})\right] \leq \bar{w}_{\text {diss }}(t)$ (recall that $\bar{w}_{\text {diss }}(t)$ is expressed in $k_{B} T$ nits) where the non-negative quantity $\mathcal{D}\left[p_{1}(\mathbf{x}) \| p_{2}(\mathbf{x})\right]:=\int d \mathbf{x} p_{1}(\mathbf{x}) \ln \left[p_{1}(\mathbf{x}) / p_{2}(\mathbf{x})\right]$ is the relative entropy, or Kullback-Leibler divergence, that measures the deviation between the two indicated distribution functions [S. Kullback, R. A. Leibler, "On information and sufficiency", Annals of Mathematical Statistics 22, 7986 (1951)].

[21] In all cases the actual state of the external agent may be specified by a set of parameters $\boldsymbol{\epsilon}(t)$ which evolve cyclically with a period $\tau_{\text {ext }}$; correspondingly, the Fourier decomposition of the energy evolution will feature a dominant frequency $\omega_{\text {ext }}=2 \pi / \tau_{\text {ext }}$ and, possibly, its integer multiples. The interaction between the agent and the system generates an energy perturbation $V_{1}(\mathbf{x}, t) \equiv F_{\text {int }}(\mathbf{x}, \boldsymbol{\epsilon}(t))$ where $F_{\text {int }}$ stands for a given functional form. Thus, the periodic evolution of the $\boldsymbol{\epsilon}(t)$ components produces a similar evolution of $V_{1}(\mathbf{x}, t)$ which, for any configuration $\mathbf{x}$, will feature a periodic trend with period $\tau=\tau_{\text {ext }} / k$ (hence a fundamental frequency $\left.\omega=k \omega_{\text {ext }}\right)$ with $k \geq 1$ an integer value. Clearly, if $F_{\text {int }}(\mathbf{x}, \boldsymbol{\epsilon})$ depends linearly on $\boldsymbol{\epsilon}$ then $k=1$, while in general cases $\omega$ may be larger than $\omega_{\text {ext }}$.

[22] L. Gammaitoni, P. Hänggi, P. Jung, and F. Marchesoni, Eur. Phys. J. B 69, 1 (2009).

[23] G. Moro and P. L. Nordio, Mol. Phys. 56, 255 (1985).

[24] R. R. Coifman, I. G. Kevrekidis, S. Lafon, M. Maggioni, and B. Nadler, Multiscale Model. Simul. 7, 842 (2008).

[25] M. A. Rohrdanz, W. Zheng, M. Maggioni, and C. Clementi, J. Chem. Phys. 134, 124116 (2011).

[26] To see this, it is sufficient to change the integration variable as $t \rightarrow u=t / \tau$; this yields $v_{n}(N)=\int_{0}^{1} d u\left\langle g_{n}(\mathbf{x})^{*} V_{1}(\mathbf{x}, t=u \tau)\right\rangle_{0} e^{-i 2 \pi N u}$. By considering that the time-dependence in $V_{1}(\mathbf{x}, t)$ must be expressed by some functional form in $t / \tau$ to be consistent with the periodicity, that is $V_{1}(\mathbf{x}, t) \equiv \tilde{V}_{1}(\mathbf{x}, t / \tau)$ and hence $V_{1}(\mathbf{x}, t=u \tau) \equiv \tilde{V}_{1}(\mathbf{x}, u)$, it follows that $v_{n}(N)$ is 
independent of $\tau$.

[27] Note that $\mathcal{D}_{\lambda_{n}}\left(\omega_{N}\right) \equiv \pi \omega_{N} \mathcal{L}_{0, \lambda_{n}}\left(\omega_{N}\right)$ where $\mathcal{L}_{x_{c}, \delta}(x)=\pi^{-1}\left[\delta^{2}+\left(x-x_{c}\right)^{2}\right]^{-1}$ stands for the Lorentzian function centered at $x=x_{c}$, of half-width at half-height equal to $\delta$, and normalized to 1 .

[28] S.-W. Wang, K. Kawaguchi, S. i. Sasa, and L.-H. Tang, arXiv:1601.04463v2 [cond-mat.statmech] (2016).

[29] By considering two state-dependent functions $f_{A}(\mathbf{x})$ and $f_{B}(\mathbf{x})$ (possibly complex-valued), their time-correlation function for a stationary Markov processes on $\mathbf{x}$ is $G_{f_{A}, f_{B}}(t):=$ $\lim _{t_{f} \rightarrow \infty} \frac{1}{t_{f}} \int_{0}^{t_{f}} d t_{0} f_{A}\left(\mathbf{x}\left(t_{0}\right)\right)^{*} f_{B}\left(\mathbf{x}\left(t_{0}+t\right)\right)$. By converting this time-average into an equivalent ensemble-average, a straightforward elaboration leads to the form $G_{f_{A}, f_{B}}(t)=$ $\int d \mathbf{x} f_{A}(\mathbf{x})^{*} e^{-t \hat{\Gamma}_{0}} p_{e q, 0}(\mathbf{x}) f_{B}(\mathbf{x})$ where $\hat{\Gamma}_{0}$ is the Smoluchowski operator. By employing the symmetrized form of such an operator, $\tilde{\Gamma}_{0}=p_{e q, 0}(\mathbf{x})^{-1 / 2} \hat{\Gamma}_{0} p_{e q, 0}(\mathbf{x})^{1 / 2}$, the latter expression becomes $G_{f_{A}, f_{B}}(t)=\int d \mathbf{x} f_{A}(\mathbf{x})^{*} p_{e q, 0}(\mathbf{x})^{1 / 2} e^{-t \tilde{\Gamma}_{0}} p_{e q, 0}(\mathbf{x})^{1 / 2} f_{B}(\mathbf{x})$.

[30] S. R. de Groot and P. Mazur, Non-equilibrium thermodynamics (Dover Publications, New York, 1984).

[31] In passing, the Onsager-like kinetic coefficients (see equations (40) with (24) of ref. [10]) are found to be expressed in terms of "generalized equilibrium correlation functions" which are defined (equation (41) of ref. [10]) by time-averages reminiscent of those which enter the definitions of our functions $\Phi_{1}$ and $\Phi_{2}$.

[32] The choice of Gaussian white noise is purely subjective. A different kind of white noise would lead to a different typology of single trajectories, but to the same ensemble properties under the sole requisite that $\eta_{t}$ is drawn from a distribution with a zero mean and unit variance.

[33] Let us consider the $x$-domain extended from $x_{\min }$ to $x_{\max }$, and make a homogeneous partition into $N_{p}$ intervals of width $\Delta x=\left(x_{\max }-x_{\min }\right) / N_{p}$. Let $x_{n}$ be the coordinate of the central point of the $n$-th interval, while $x_{n}^{+} \equiv x_{n+1}^{-}$and $x_{n}^{-} \equiv$ $x_{n-1}^{+}$are the extrema of such an interval. Then we set $g(x, t)=e^{-V(x, t)}$. The discretization route applied to the Fokker-Planck-Smoluchowski equation with constant diffusion coefficient $D$ leads to $M_{n m}(t)=\delta_{n, m} D(\Delta x)^{-2} g\left(x_{n}, t\right)^{-1}\left[g\left(x_{n}^{+}, t\right)+g\left(x_{n}^{-}, t\right)\right]-$ $\delta_{n+1, m} D(\Delta x)^{-2} g\left(x_{n+1}, t\right)^{-1} g\left(x_{n}^{+}, t\right)-\delta_{n-1, m} D(\Delta x)^{-2} g\left(x_{n-1}, t\right)^{-1} g\left(x_{n}^{-}, t\right)$ with the following specific elements imposed by periodic boundary conditions (i.e., the equality of the probability-density fluxes, generally non-null, at the boundaries): $M_{1 N_{p}}(t)=$ $-D(\Delta x)^{-2} g\left(x_{N_{p}}, t\right)^{-1} g\left(x_{1}^{-}, t\right)$ and $M_{N_{p} 1}(t)=-D(\Delta x)^{-2} g\left(x_{1}, t\right)^{-1} g\left(x_{N_{p}}^{+}, t\right)$. In the stationary case (absence of external time-dependent perturbation), the matrix $\mathbf{M}(t)$ reduces to $\mathbf{M}_{0}$. The equations above still hold with $g_{0}(x)=e^{-V_{0}(x)}$ in place of $g(x, t)$. In such a situation, the balancing $\left[\tilde{\mathbf{M}}_{0}\right]_{n m}=\left[\mathbf{M}_{0}\right]_{n m}\left[g_{0}\left(x_{m}\right) / g_{0}\left(x_{n}\right)\right]^{1 / 2}$ yields the real-symmetric matrix $\tilde{\mathbf{M}}_{0}$ which 
corresponds to the symmetrized operator $\tilde{\Gamma}_{0}=p_{e q, 0}(x)^{-1 / 2} \hat{\Gamma}_{0} p_{e q, 0}(x)^{1 / 2}$.

[34] First of all, for both cases the energy perturbation is written as $V_{1}(x, t)=$ $\epsilon(t)[\cos x \cos \varphi(\epsilon(t))-\sin x \sin \varphi(\epsilon(t))]$, with $\varphi(\epsilon(t))=0$ in Case 1 and $\varphi(\epsilon(t))=\phi \epsilon(t) / \Delta$ in Case 2. For the sake of compactness, let us introduce the following integrals: $I_{c}(n)=$ $\int d x \cos x \phi_{n}(x) \phi_{0}(x)$ and $I_{s}(n)=\int d x \sin x \phi_{n}(x) \phi_{0}(x)$ where $\phi_{n}(x)$ are the eigenfunctions (here real-valued) of the symmetrized Smoluchowski operator $\tilde{\Gamma}_{0}$. Moreover, $K_{1}(N)=$ $\tau^{-1} \int_{0}^{\tau} d t \epsilon(t) \cos \left(\varphi(\epsilon(t)) \cos (N \omega t), K_{2}(N)=\tau^{-1} \int_{0}^{\tau} d t \epsilon(t) \sin \left(\varphi(\epsilon(t)) \cos (N \omega t), K_{3}(N)=\right.\right.$ $\tau^{-1} \int_{0}^{\tau} d t \epsilon(t) \cos \left(\varphi(\epsilon(t)) \sin (N \omega t)\right.$ and $K_{4}(N)=\tau^{-1} \int_{0}^{\tau} d t \epsilon(t) \sin (\varphi(\epsilon(t)) \sin (N \omega t)$. In Case 1, the integration over the time variable in Eq. 21 is analytical and few steps lead to obtain $\left|v_{n}(1)\right|^{2}=(\Delta / 2)^{2}\left|I_{c}(n)\right|^{2}$ and $\left|v_{n}(N)\right|^{2}=0$ for $N \neq 1$. In Case 2, some elaborations yield $\left|v_{n}(N)\right|^{2}=\left[I_{c}(n) K_{1}(N)-I_{s}(n) K_{2}(N)\right]^{2}+\left[I_{c}(n) K_{3}(N)-I_{s}(n) K_{4}(N)\right]^{2}$. The computation of the integrals in $I_{c}(n)$ and $I_{s}(n)$ is made by employing the rectangles rule and by using the eigenvectors of the matrix $\tilde{\mathbf{M}}_{0}$. Namely, if $\tilde{\mathbf{M}}_{0} \mathbf{E}=\mathbf{E} \boldsymbol{\Lambda}$, with $\mathbf{E}$ the matrix whose columns are the normalized eigenvectors (i.e., $\sum_{n^{\prime}=0}^{N_{p}} E_{n n^{\prime}}^{2}=1$ for any $n$ ) and $\boldsymbol{\Lambda}$ the diagonal matrix of the eigenvalues, then $I_{c}(n) \simeq \sum_{n^{\prime}=0}^{N_{p}} \cos x_{n} E_{n n^{\prime}} E_{0 n^{\prime}}$ and $I_{s}(n) \simeq \sum_{n^{\prime}=0}^{N_{p}} \sin x_{n} E_{n n^{\prime}} E_{0 n^{\prime}}$. The integrals $K_{1}(N)$ to $K_{4}(N)$ are computed here by adopting a library routine which employs Romberg integration.

[35] S. T. Milner and S. A. Safran, Phys. Rev. A 36, 4371 (1987).

[36] E. Sackmann, 'Physical basis of self-organization and function of membranes: physics of vesicles', Chapter 5 in 'Structure and dynamics of membranes - Vol. 1 A - From cells to vesicles' (R. Lipowsky and E. Sackmann editors) (1995, Elsevier, Amsterdam).

[37] W. Helfrich, Z. Naturforsch 29c, 182 (1974).

[38] M. D. Mitov, P. Méléard, M. Winterhalter, M. I. Angelova, and P. Bothorel, Phys. Rev. E 48, 628 (1993).

[39] W. Helfrich, Z. Naturforsch 28c, 693 (1973).

[40] R. Dimova, K. A. Riske, S. Aranda, N. Bezlyepkina, R. L. Knorr, and R. Lipowsky, Soft Matter 3, 817 (2007).

[41] The case of AC electric fields would pose a number of interesting issues when the field frequency is small enough (roughly, in the sub-kHz regime) since the perturbation induces a frequencydependent dynamic response of the internal and external fluid media depending on their conductivities (see refs. [38] and [40]). For this kind of perturbation, the description of the system should comprise degrees of freedom of the fluid media.

[42] T. Schmiedl and U. Seifert, Phys. Rev. Lett. 98, 108301 (2007).

[43] The practical motivation is to reduce as much as possible the error on free energy differences estimated from measures of the work amounts in repeated transformations. $[3,5]$. 
[44] To prove Eq. A3, let us start by considering that $\delta V_{1}(\mathbf{x}, t)=V_{1}(\mathbf{x}, t)-\left\langle V_{1}(\mathbf{x}, t)\right\rangle_{0}=$ $\sum_{N=-\infty}^{+\infty} \sum_{n \geq 1} v_{n}(N) g_{n}(\mathbf{x}) e^{i N \omega t} \quad$ where the functions $g_{n}(\mathbf{x})$ have been introduced in Section II A; the contribution for $n=0$ is excluded since it corresponds precisely to $\left\langle V_{1}(\mathbf{x}, t)\right\rangle_{0}$. By employing such an expression to expand $\delta V_{1}\left(\mathbf{x}, t^{\prime}\right)$ and $\delta V_{1}\left(\mathbf{x}, t^{\prime \prime}\right)$ in Eq. A1 (and taking the complex-conjugation for $\delta V_{1}\left(\mathbf{x}, t^{\prime}\right)$ without altering the result since such a function is real-valued), it follows $G_{\delta V_{1}\left(t^{\prime}\right), \delta V_{1}\left(t^{\prime \prime}\right)}(t)=\sum_{N_{1}, N_{2}=-\infty}^{+\infty} \sum_{n, n^{\prime} \geq 1} v_{n}\left(N_{1}\right)^{*} v_{n^{\prime}}\left(N_{2}\right) e^{i \omega\left(-N_{1} t^{\prime}+N_{2} t^{\prime \prime}\right)} G_{g_{n}, g_{n^{\prime}}}(t)$ where $G_{g_{n}, g_{n^{\prime}}}(t)$ is the time-correlation function (see note [29]) between $g_{n}(\mathbf{x})$ and $\quad g_{n^{\prime}}(\mathbf{x}): \quad G_{g_{n}, g_{n^{\prime}}}(t)=\int d \mathbf{x} g_{n}(\mathbf{x})^{*} p_{e q, 0}(\mathbf{x})^{1 / 2} e^{-t \tilde{\Gamma}_{0}} p_{e q, 0}(\mathbf{x})^{1 / 2} g_{n^{\prime}}(\mathbf{x})$. Since $\phi_{n}(\mathbf{x})=g_{n}(\mathbf{x}) p_{e q, 0}(\mathbf{x})^{1 / 2}$ are ortho-normal eigenfunctions of the symmetrized operator $\tilde{\Gamma}_{0}$ with eigenvalues $\lambda_{n}$, one has that $G_{g_{n}, g_{n^{\prime}}}(t)=\delta_{n, n^{\prime}} e^{-\lambda_{n} t}$ and hence $G_{\delta V_{1}\left(t^{\prime}\right), \delta V_{1}\left(t^{\prime \prime}\right)}(t)=\sum_{N_{1}, N_{2}=-\infty}^{+\infty} \sum_{n \geq 1} v_{n}\left(N_{1}\right)^{*} v_{n}\left(N_{2}\right) e^{i \omega\left(-N_{1} t^{\prime}+N_{2} t^{\prime \prime}\right)} e^{-\lambda_{n} t}$. Multiplication at both members by $\sigma\left(t^{\prime}, t^{\prime \prime}, t\right)$ defined in Eq. A4 yields $G_{\delta V_{1}\left(t^{\prime}\right), \delta V_{1}\left(t^{\prime \prime}\right)}(t) \sigma\left(t^{\prime}, t^{\prime \prime}, t\right)=$ $\sum_{N_{1}, N_{2}, N=-\infty}^{+\infty} \sum_{n \geq 1} N^{2} v_{n}\left(N_{1}\right)^{*} v_{n}\left(N_{2}\right) e^{-\lambda_{n} t} e^{-i \omega\left(N_{1}+N\right) t^{\prime}} e^{i \omega\left(N_{2}+N\right) t^{\prime \prime}} e^{-i \omega N t}$. When integrating over $t^{\prime}$ and $t^{\prime \prime}$, the complex factors $e^{-i \omega\left(N_{1}+N\right) t^{\prime}}$ and $e^{i \omega\left(N_{2}+N\right) t^{\prime \prime}}$ give, respectively, $(2 \pi / \omega) \delta_{N_{1},-N}$ and $(2 \pi / \omega) \delta_{N_{2},-N}$. Thus, $\int_{0}^{\tau} d t^{\prime} \int_{0}^{\tau} d t^{\prime \prime} G_{\delta V_{1}\left(t^{\prime}\right), \delta V_{1}\left(t^{\prime \prime}\right)}(t) \sigma\left(t^{\prime}, t^{\prime \prime}, t\right)=$ $(2 \pi / \omega)^{2} \sum_{N=-\infty}^{+\infty} \sum_{n \geq 1} N^{2} v_{n}(-N)^{*} v_{n}(-N) e^{-\lambda_{n} t} e^{-i \omega N t} . \quad$ By $\quad$ taking into account that $\lambda_{n}>0$ for $n \geq 1$, the integration on $t$ from $\quad 0 \quad$ to $\quad \infty \quad$ gives $\quad \int_{0}^{\infty} d t \int_{0}^{\tau} d t^{\prime} \int_{0}^{\tau} d t^{\prime \prime} G_{\delta V_{1}\left(t^{\prime}\right), \delta V_{1}\left(t^{\prime \prime}\right)}(t) \sigma\left(t^{\prime}, t^{\prime \prime}, t\right)=$ $(2 \pi / \omega)^{2} \sum_{N=-\infty}^{+\infty} \sum_{n \geq 1}\left(\lambda_{n}+i \omega N\right)^{-1}\left|N v_{n}(N)\right|^{2}$. The expression of $\bar{w}_{\text {diss }}^{\infty}$ given in Eq. 21 is finally obtained by taking the real part of this expression (the imaginary part results to be null since the terms $\pm N$ cancel, and the term for $N=0$ is null) and multiplying by $2 \pi \omega / \tau^{2}$. This proves Eq. A3.

[45] The proof is straightforward by starting from $v(0, \mathbf{x})=\sum_{n} v_{n}(0) g_{n}(\mathbf{x})=\tau^{-1} \int_{0}^{\tau} d t V_{1}(\mathbf{x}, t)$ according to Eqs. 15 and 16. Then it follows the identity $\tau^{-1} \int_{0}^{\tau} d t \delta V_{1}(\mathbf{x}, t)=\sum_{n \geq 1} v_{n}(0) g_{n}(\mathbf{x})$. By considering that $\chi_{b}(t)$ is nothing but the time-correlation function of the term at the lefthand side of this identity (evaluated at times $t^{\prime}$ and $t^{\prime \prime}$ ), using the form the right-hand side to expand the time-correlation (see note [29]) it follows $\chi_{b}(t)=\sum_{n, n^{\prime} \geq 1} v_{n}(0)^{*} v_{n^{\prime}}(0) G_{g_{n}, g_{n^{\prime}}}(t)=$ $\sum_{n \geq 1}\left|v_{n}(0)\right|^{2} e^{-\lambda_{n} t}$ Then, $d \chi_{b}(t) /\left.d t\right|_{t=0}=-\sum_{n \geq 1} \lambda_{n}\left|v_{n}(0)\right|^{2}$.

[46] A. Imparato, L. Peliti, G. Pesce, G. Rusciano, and A. Sasso, Phys. Rev. E 76, 050101 (2007).

[47] A possible route is sketched hereafter. By tracing the work of Imparato et al. [46], let us introduce the joint probability distribution $\Psi(\mathbf{x}, w, t)$ with marginal distributions $p(\mathbf{x}, t)=$ $\int d w \Psi(\mathbf{x}, w, t)$ and $p(w, t)=\int d \mathbf{x} \Psi(\mathbf{x}, w, t)$ (here, $p(w, t)$ is the distribution function of the work performed up to time $t$ starting from a configuration sampled from the equilibrium distribution $\left.p_{e q, 0}(\mathbf{x})\right)$. The conditions at boundaries are $\lim _{|w| \rightarrow \infty} \Psi(\mathbf{x}, w, t)=0$ for any $\mathbf{x}$ and 
$t$. For the specific initial state considered here (before the perturbation starts), the initial condition is $\Psi(\mathbf{x}, w, 0)=p_{e q, 0}(\mathbf{x}) \delta(w)$ with $\delta$ denoting here, and below, the Dirac's Delta function. The partial differential equation (PDE) which specifies the evolution of $\Psi$ is derived in ref. [46]. In our notation it reads $\partial \Psi(\mathbf{x}, w, t) / \partial t=-\left[\hat{\Gamma}(t)+\left(\partial V_{1}(\mathbf{x}, t) / \partial t\right) \partial / \partial w\right] \Psi(\mathbf{x}, w, t)$. Then, let us consider the two-times conditional probability distribution $\Psi_{2}\left(\mathbf{x}, w, t \mid \mathbf{x}_{0}, w_{0}, t_{0}\right)$ with $\Psi_{2}\left(\mathbf{x}, w, t_{0} \mid \mathbf{x}_{0}, w_{0}, t_{0}\right)=\delta\left(\mathbf{x}-\mathbf{x}_{0}\right) \delta\left(w-w_{0}\right)$. The evolution of $\Psi_{2}$ is specified by the same PDE as for $\Psi$. The solution of these PDEs with the given initial conditions yields the two distributions. Using the law of probability composition makes it possible to express the probability distribution $p_{t_{0} \rightarrow t_{1}}(w)$ for the work performed in the interval between $t_{0}$ and $t_{1}$ as $p_{t_{0} \rightarrow t_{1}}(w)=\int d \mathbf{x}_{0} \int d \mathbf{x}_{1} \int d w_{0} \int d w_{1} \Psi\left(\mathbf{x}_{0}, w_{0}, t_{0}\right) \Psi_{2}\left(\mathbf{x}_{1}, w_{1}, t_{1} \mid \mathbf{x}_{0}, w_{0}, t_{0}\right) \delta\left(w_{1}, w_{0}+w\right)$. Application of the property of the Delta function in the integration finally gives $p_{t_{0} \rightarrow t_{1}}(w)=$ $\int d \mathbf{x}_{0} \int d \mathbf{x}_{1} \int d w_{0} \Psi\left(\mathbf{x}_{0}, w_{0}, t_{0}\right) \Psi_{2}\left(\mathbf{x}_{1}, w_{0}+w, t_{1} \mid \mathbf{x}_{0}, w_{0}, t_{0}\right)$. Then one should consider $t_{0} \equiv$ $t_{n_{c}-1}$ and $t_{1}=t_{n_{c}}$ to obtain the work distribution for the $n_{c^{-}}$th cycle.

[48] To construct $p\left(w_{\text {diss }}^{\infty}\right)$, an ensemble of $w_{\text {diss }}^{\infty}$ values has been generated by producing a long stochastic trajectory under perturbation. It would be interesting to analyse, which we will leave for future studies, the extent of $\tau$-dependent correlations between the values of such a sequence.

[49] A rationale is the following. Let us recall the work distribution function $p(w, t)$ and the joint probability distribution $\Psi(\mathbf{x}, w, t)$ already introduced in the note [47], with $p(w, t)=\int d \mathbf{x} \Psi(\mathbf{x}, w, t)$. From the rule of probability composition, it follows (i) $p\left(w, t_{n_{c}}\right)=$ $\int d w_{n_{c}} \int d \mathbf{x} \Psi\left(\mathbf{x}, w-w_{n_{c}}, t_{n_{c}-1}\right) \rho_{n_{c}}\left(w_{n_{c}} \mid \mathbf{x}, w-w_{n_{c}}, t_{n_{c}-1}\right)$, where $\rho_{n_{c}}\left(w_{n_{c}} \mid \mathbf{x}, w-w_{n_{c}}, t_{n_{c}-1}\right)$ is the conditioned probability to perform a quantity of work $w_{n_{c}}$ during the $n_{c}$-th cycle if the work done up to time $t_{n_{c}-1}$ is equal to $w-w_{n_{c}}$ and the system's configuration is $\mathbf{x}$ at that instant. The key-assumption is that the dependence on the state at time $t_{n_{c}-1}$ becomes weaker and weaker as the number of performed cycles increases, that is, (ii) $\rho_{n_{c}}\left(w_{n_{c}} \mid \mathbf{x}, w-w_{n_{c}}, t_{n_{c}-1}\right) \rightarrow p_{\infty}\left(w_{n_{c}}\right)$ for $n_{c} \rightarrow \infty$ where $p_{\infty}\left(w_{n_{c}}\right)$ stands for the limit distribution. By recalling that the work per cycle is entirely dissipated, such a limit distribution corresponds to $p\left(w_{\text {diss }}^{\infty}\right)$. As consequence, the integration over $\mathbf{x}$ in Eq. (i) implies that (iii) $p\left(w, t_{n_{c}}\right) \simeq \int d w_{\text {diss }}^{\infty} p\left(w-w_{\text {diss }}^{\infty}, t_{n_{c}-1}\right) p\left(w_{\text {diss }}^{\infty}\right)$ for $n_{c} \rightarrow \infty$. By multiplying both members by $e^{-w}$ and integrating over $w$, the application of Jarzynski's equality (JE) [17] under consideration that the system's free energy difference is null at completion of any number of cycles, yields $\int d w p\left(w, t_{n_{c}}\right) e^{-w} \stackrel{\mathrm{JE}}{=} 1 \simeq \int d w \int d w_{\text {diss }}^{\infty} p\left(w-w_{\text {diss }}^{\infty}, t_{n_{c}-1}\right) e^{-w} p\left(w_{\text {diss }}^{\infty}\right)$. The switch of the order

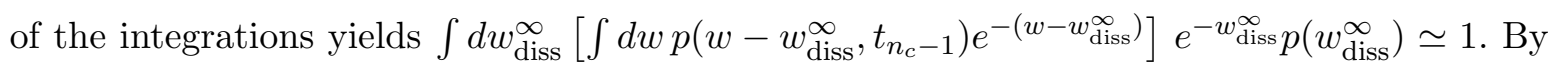
making the change of integration variable $w \rightarrow w^{\prime}=w-w_{\text {diss }}^{\infty}$, the JE leads to recognize that the integral within square brackets is equal to 1 (integration boundaries are kept at $\pm \infty$ ). 
Thus, it follows the integral constraint (iv) $\int d w_{\text {diss }}^{\infty} e^{-w_{\text {diss }}^{\infty}} p\left(w_{\text {diss }}^{\infty}\right) \simeq 1$. [In passing, note that this constraint is compatible with the approximate fulfillment of the SSFT relation (Appendix B) once it is written as $p\left(-w_{\text {diss }}^{\infty}\right) \simeq e^{-w_{\text {diss }}^{\infty}} p\left(w_{\text {diss }}^{\infty}\right)$ and the integration on $w_{\text {diss }}^{\infty}$ is performed at both members.] The decoupling condition Eq. (ii), intuitively, should hold if the period $\tau$ is much longer than the intrinsic fluctuation times of the system at the underlying equilibrium; for small perturbations, these times can be taken to be the inverse of the characteristic fluctuation frequencies at the unperturbed equilibrium (see Section II A). For such a slow energy modulation, the configuration-dependent lag between $p(\mathbf{x}, t)$ and $p_{e q, t}(\mathbf{x})$ remains small from time-zero on, and asymptotically tends to attain a limit as the number of cycles increases. In these conditions it is reasonable to assume that $p\left(w, t_{n_{c}}\right)$ and $p\left(w, t_{n_{c}-1}\right)$ are well approximate by Gaussian functions whose shift and broadening, cycle by cycle, are regulated by $\bar{w}_{\text {diss }}^{\infty}$. Hence, the convolution-like operation in Eq. (iii) suggests that also $p\left(w_{\text {diss }}^{\infty}\right)$ may be close to a Gaussian distribution. The integral constraint in Eq. (iv) then implies $\sigma_{\infty}^{2}=2 \bar{w}_{\text {diss }}^{\infty}$ about the variance. As a whole, the Gaussian profile is expected in the long- $\tau$ limit.

[50] A survey of the literature gives us the idea that the soundness of the SSFT accuracy is still under debate, and that the fulfillment of such a relation seems to depend in a rather intricate way by the interplay between the relaxation properties of the unperturbed system and the nature of the perturbation. For overdamped dynamics such as those considered in our study, the soundness of the SSFT over only one cycle in the asymptotic limit has been proven both experimentally and numerically in a number of case models $[4,7]$, although it has been argued that it should be satisfied only for "linear systems" [see B. Saha and S. Mukherji, J. Stat. Mech. p. P08014 (2014)] which, in our terminology, correspond to landscapes of $V_{0}(\mathbf{x})$ featuring a single multidimensional parabolic well. Deviations are recognized for non-linear systems in the underdamped regime of motion [see M. I. Dykman, Phys. Rev. E 77, 021123 (2008); P. Zhou, X. Dong, C. Stambaugh and H. B. Chan, Phys. Rev. E 91, 052110 (2015)]. 


\section{CAPTIONS}

\section{Figure 1}

(a) A pictorial representation of the fluctuating system initially at equilibrium and then cyclically perturbed by an external agent. (b) The interrelations investigated in the present study.

\section{Figure 2}

Energetics for the uni-dimensional case model. (a) Energy profile of the unperturbed system; (b) contour plots showing the time-modulation of the energy profile under perturbation in Case 1 and Case 2.

\section{Figure 3}

Examples of Langevin trajectories for the unperturbed system (a) and for the perturbed system in Case 1 (b) and Case 2 (c) with period $\tau=5$.

\section{Figure 4}

Dependence of the average dissipated energy per cycle, $\bar{w}_{\text {diss }, n_{c}}$, versus the number of performed cycles for the uni-dimensional system in Case 1 and Case 2. The profiles refer to the period $\tau=5$.

\section{Figure 5}

Dependence of the asymptotic average dissipated energy per cycle, $\bar{w}_{\text {diss }}^{\infty}$, versus the frequency $\omega$ for the uni-dimensional system in Case 1 and Case 2. Solid lines refer to the approximate solution Eq. 21, while the circles refer to the exact values from the numerical solution of the non-stationary Fokker-Planck-Smoluchowski equation. The bars on the top axes correspond to the first 30 eigenvalues of the Smoluchowski operator for the unperturbed system. For Case 2, the dashed line in the low-frequency range indicates the linear growth on $\omega$.

\section{Figure 6}


Distribution functions of the dissipated energy (work) per cycle, in the asymptotic limit, for the uni-dimensional system in Case 1 and Case 2. The panels refer to different frequencies $\omega$, as indicated. The circles display the distributions constructed by histograms from a long Langevin simulation. The solid lines are the Gaussian distributions parametrized by $\bar{w}_{\text {diss }}^{\infty}$ obtained from approximate expression in Eq. 21 (see the text for details).

\section{Figure 7}

Same as for Figure 6, but here for Case 2.

\section{Figure 8}

Plot of $\ln p\left(w_{\text {diss }}^{\infty}\right) / p\left(-w_{\text {diss }}^{\infty}\right)$ vs. $w_{\text {diss }}^{\infty}$ for the uni-dimensional system in Case 1. The circles correspond to points obtained from the distributions constructed by histograms (see Figure 6). The dashed straight lines have a unitary slope.

\section{Figure 8}

Plot of $\ln p\left(w_{\text {diss }}^{\infty}\right) / p\left(-w_{\text {diss }}^{\infty}\right)$ vs. $w_{\text {diss }}^{\infty}$ for the uni-dimensional system in Case 2. The circles correspond to points obtained from the distributions constructed by histograms (see Figure 7). The dashed straight lines have a unitary slope. 

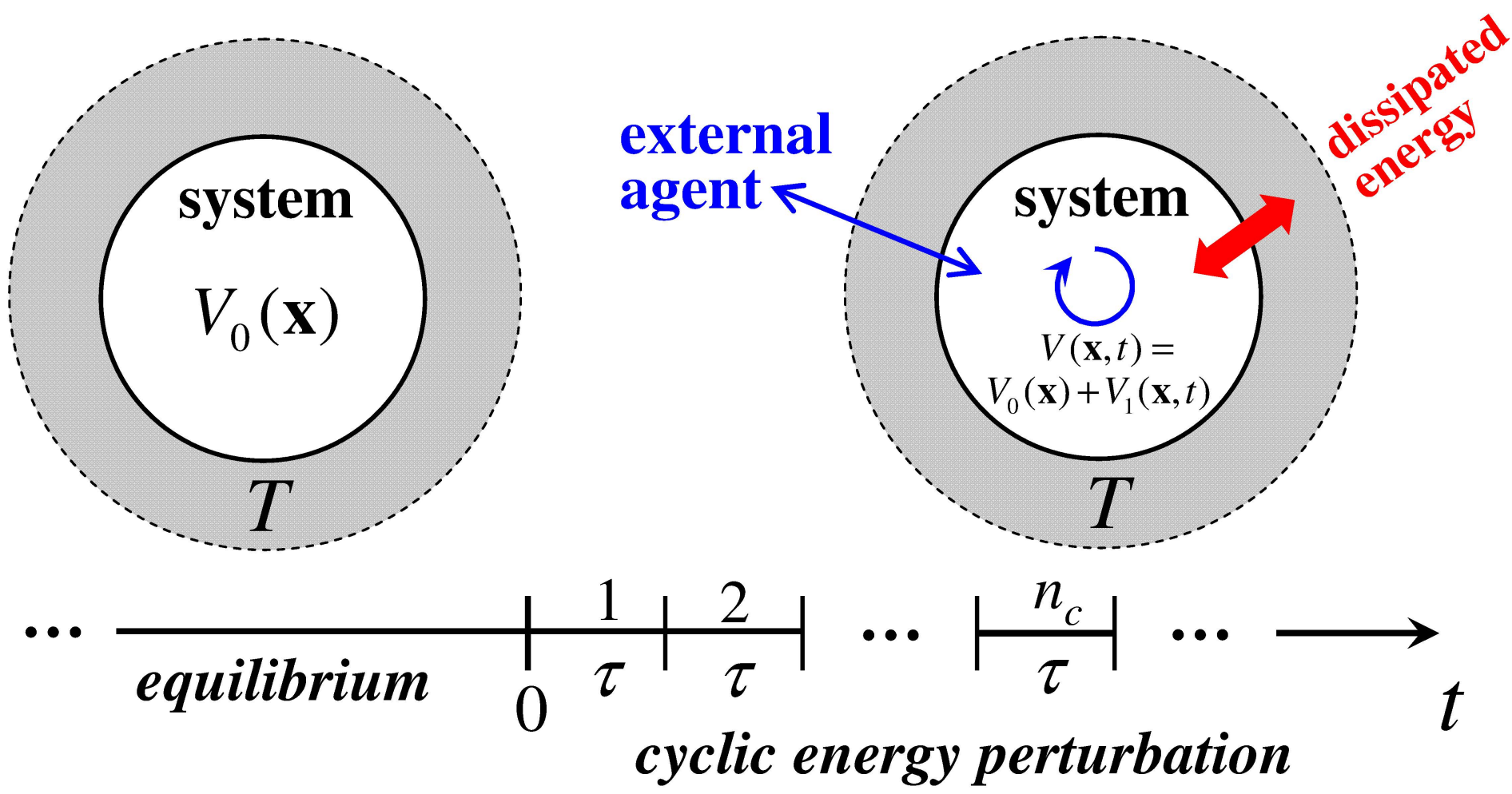

(b)

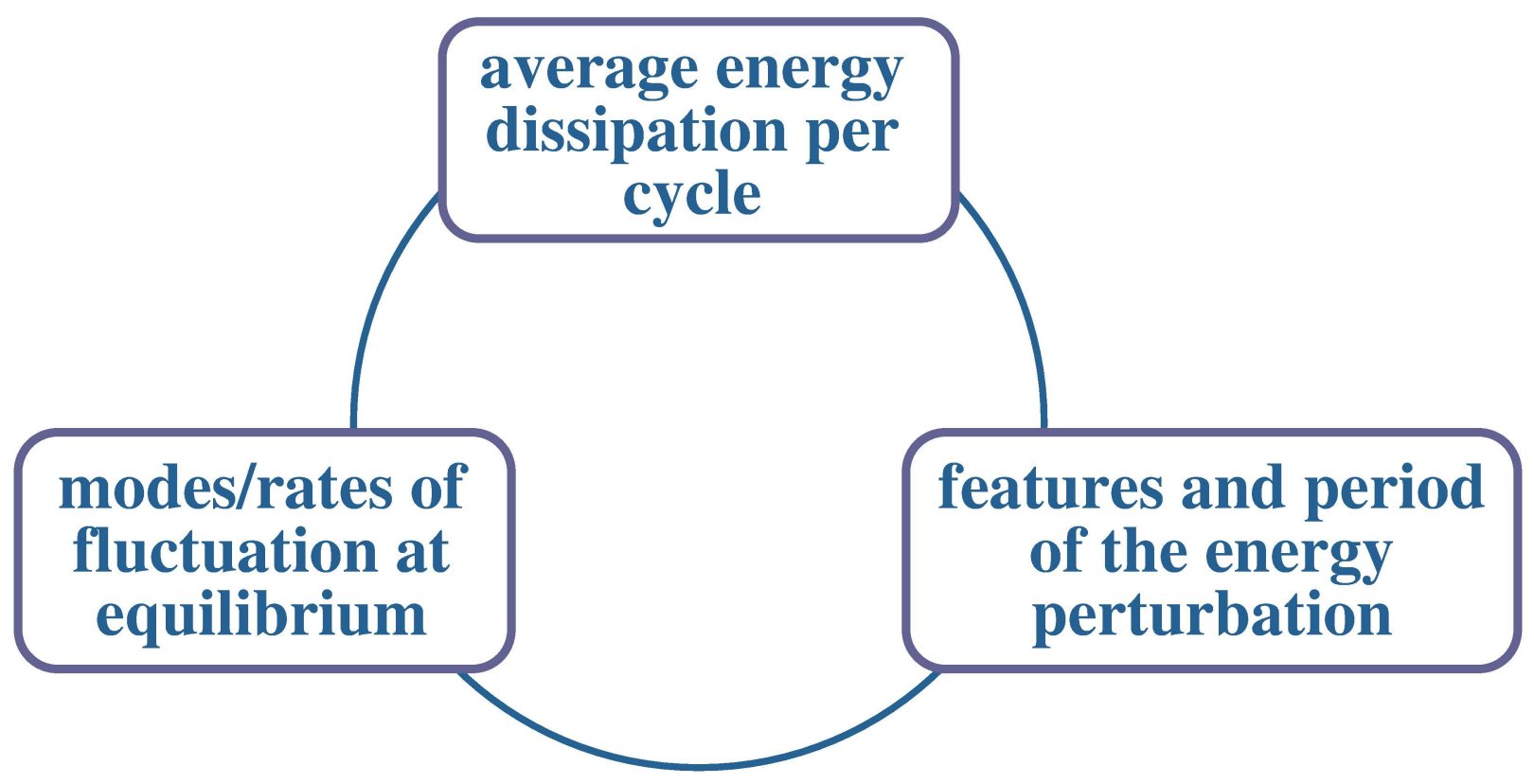




\section{(b) $\quad V(x, t)$}

\section{Case 1}

(a)
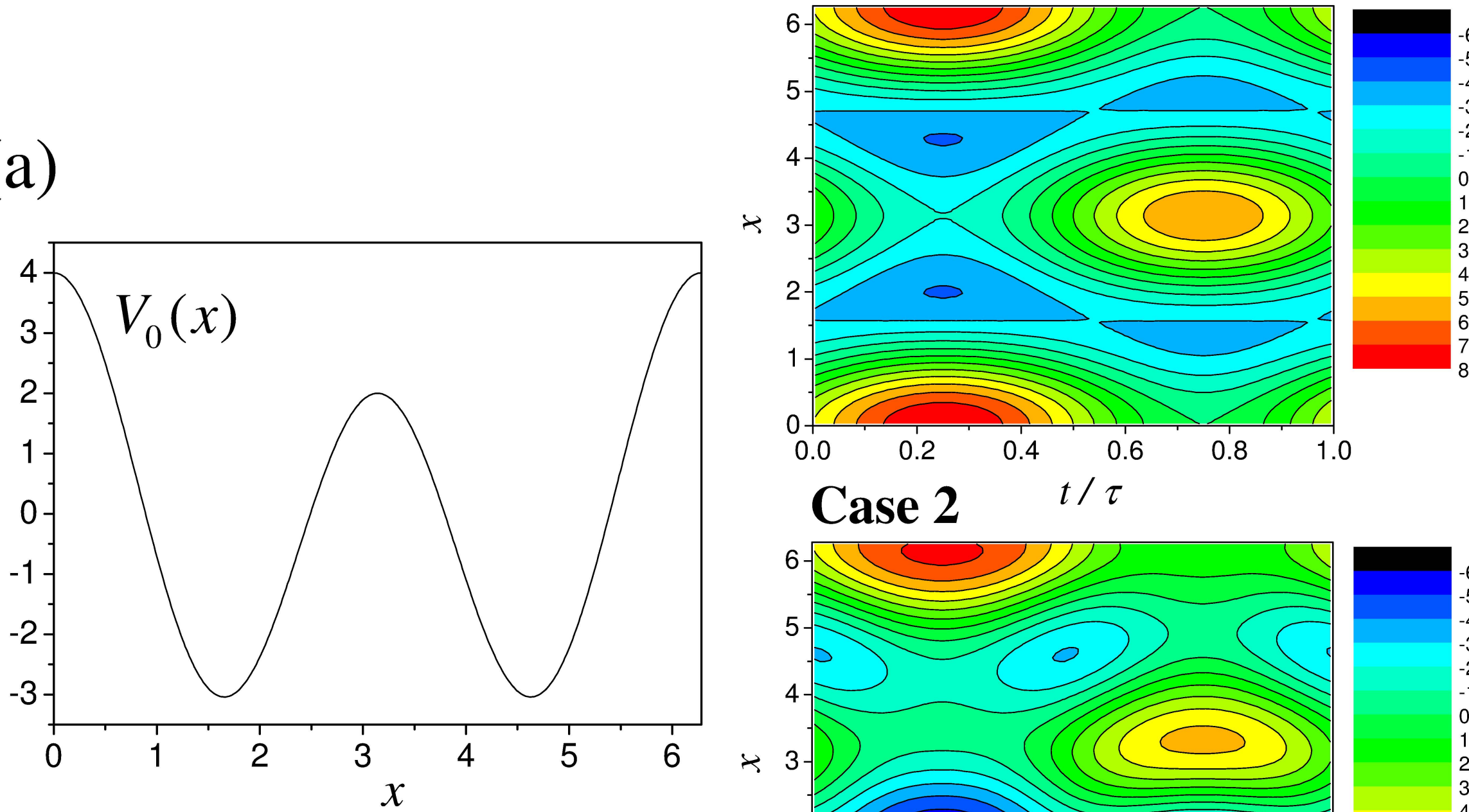

\section{Case $2 \quad t / \tau$}
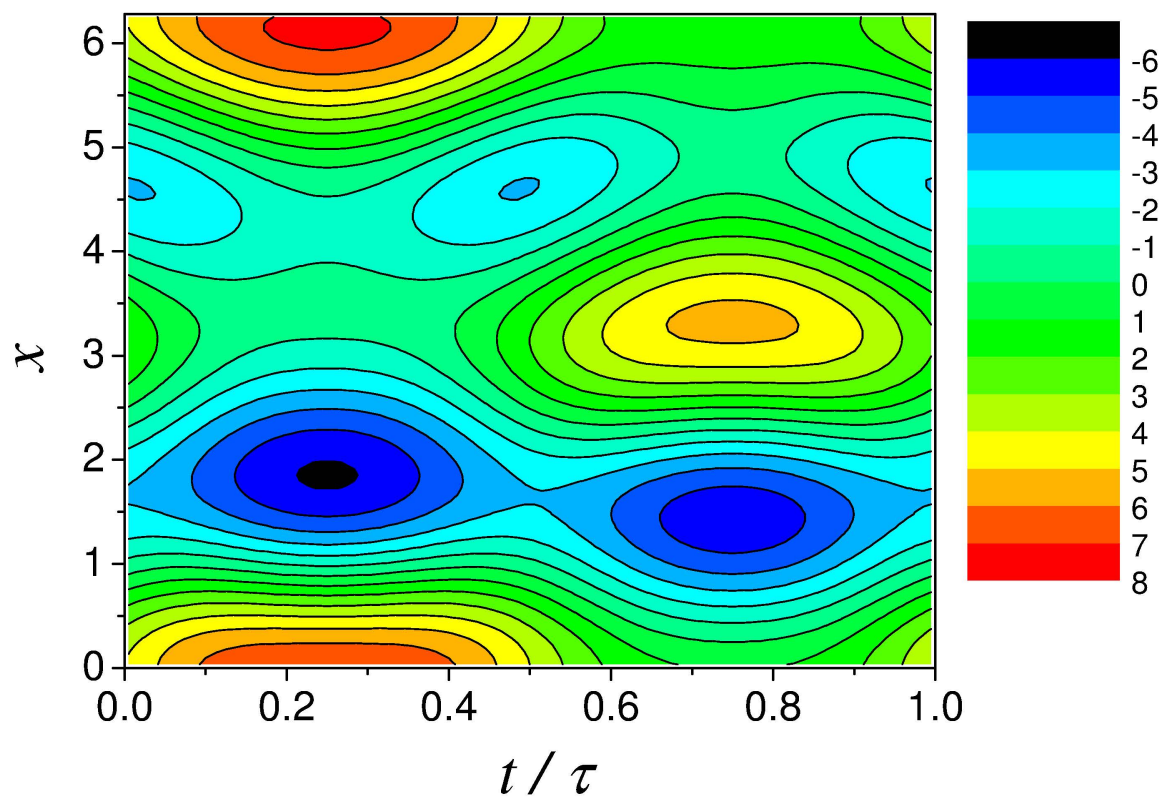
(a)

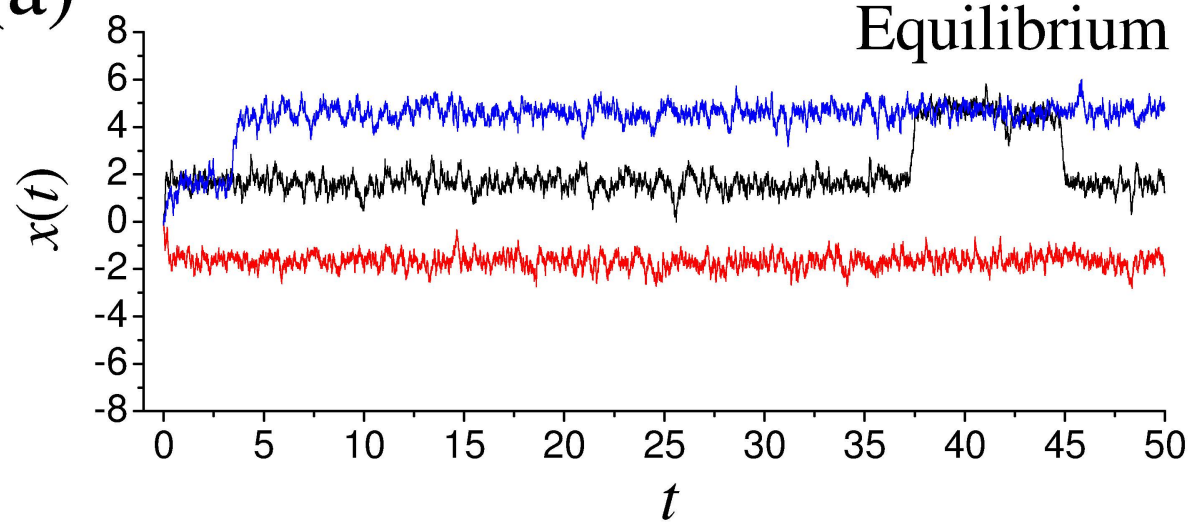

(b)

Case $1, \tau=5$

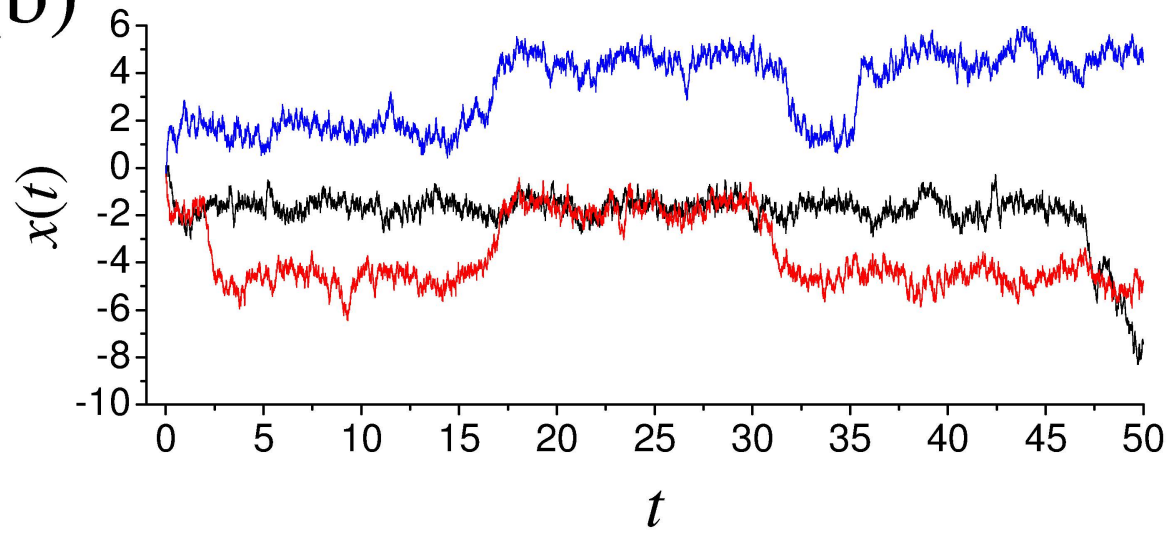

(c)

Case 2, $\tau=5$

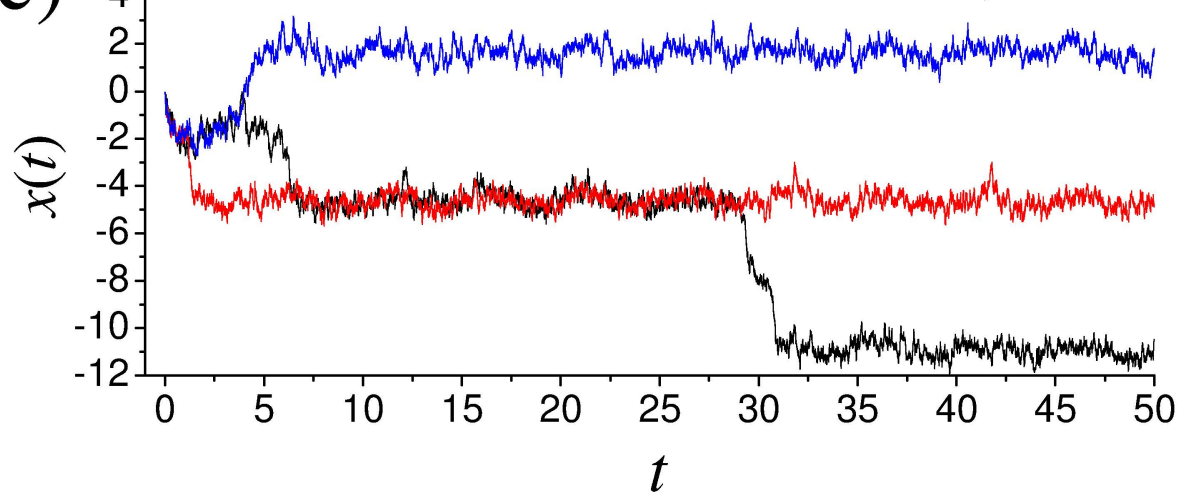




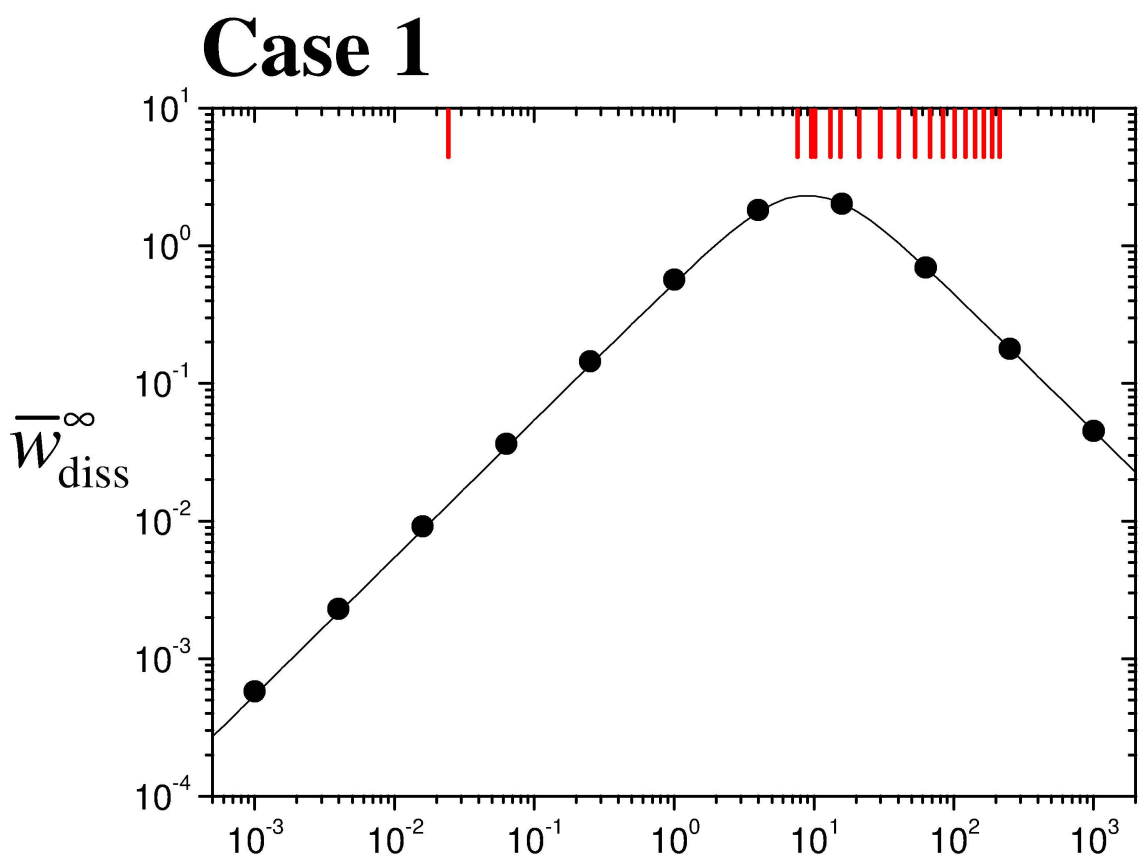

$\omega$

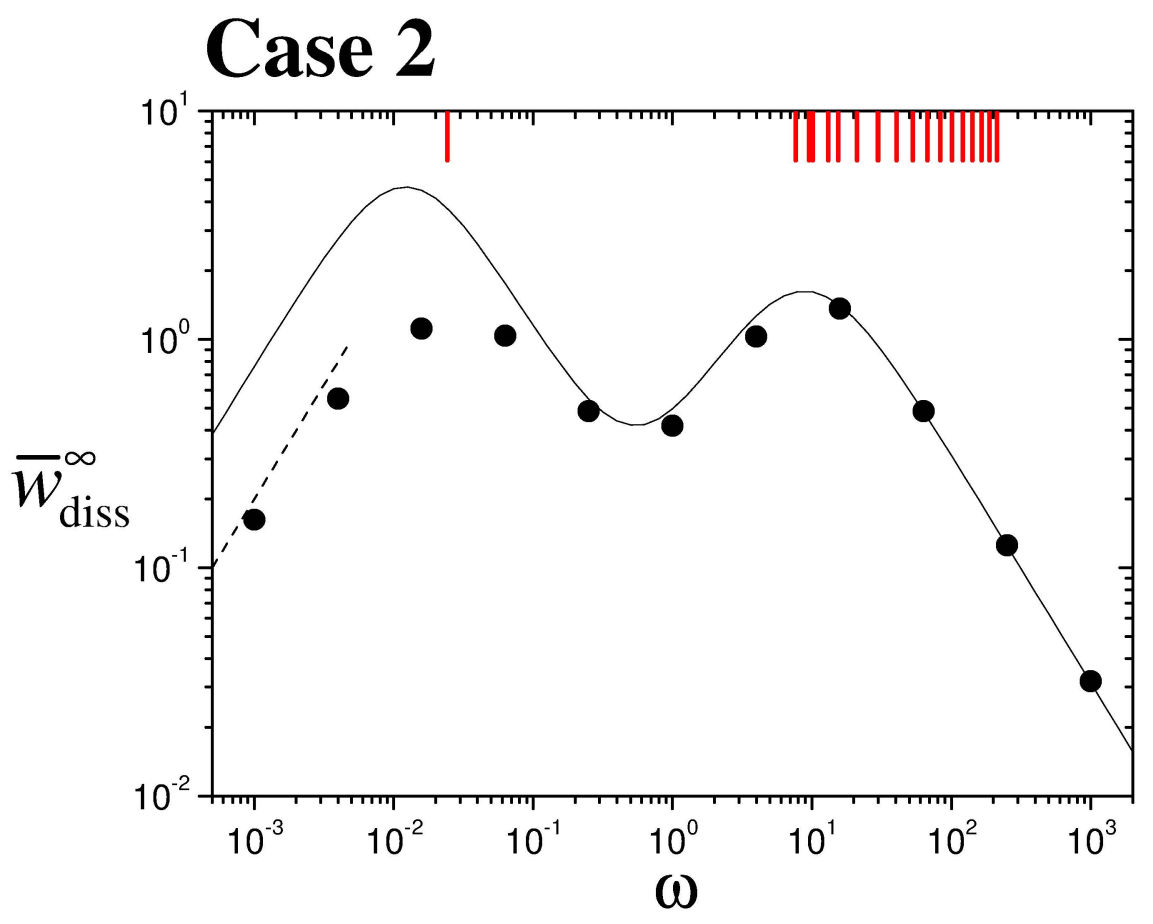



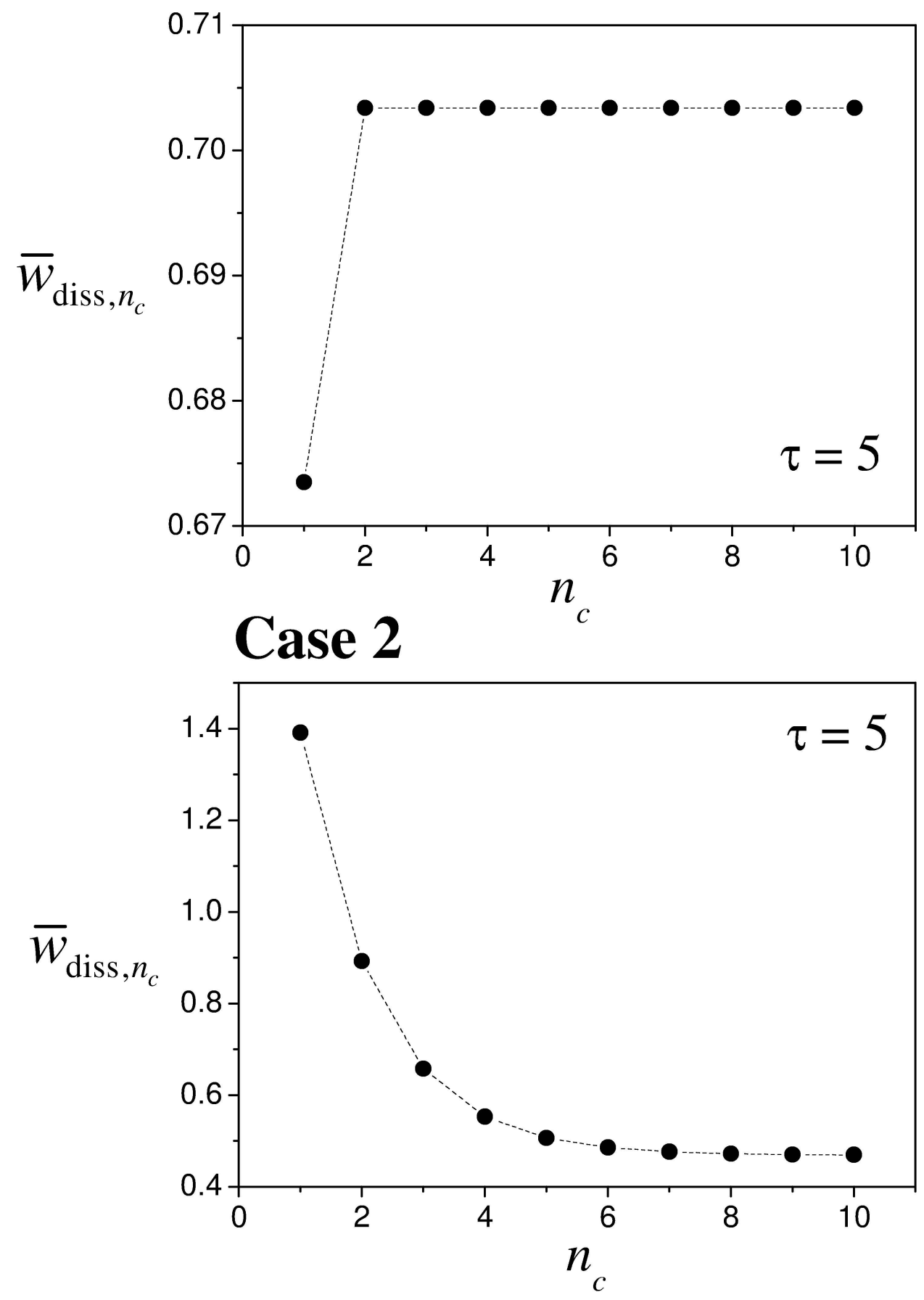


\section{Case 1}
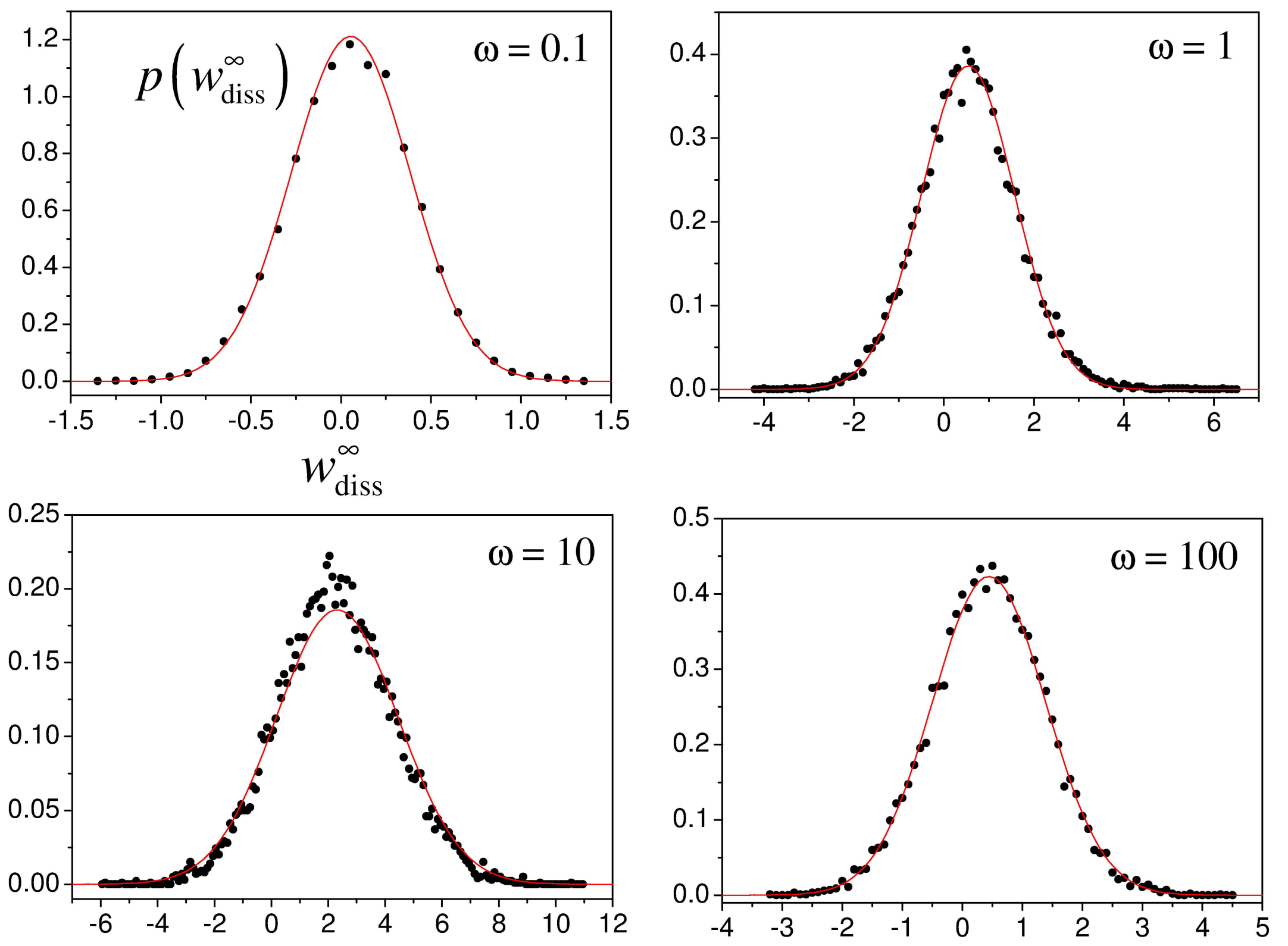
Case 2
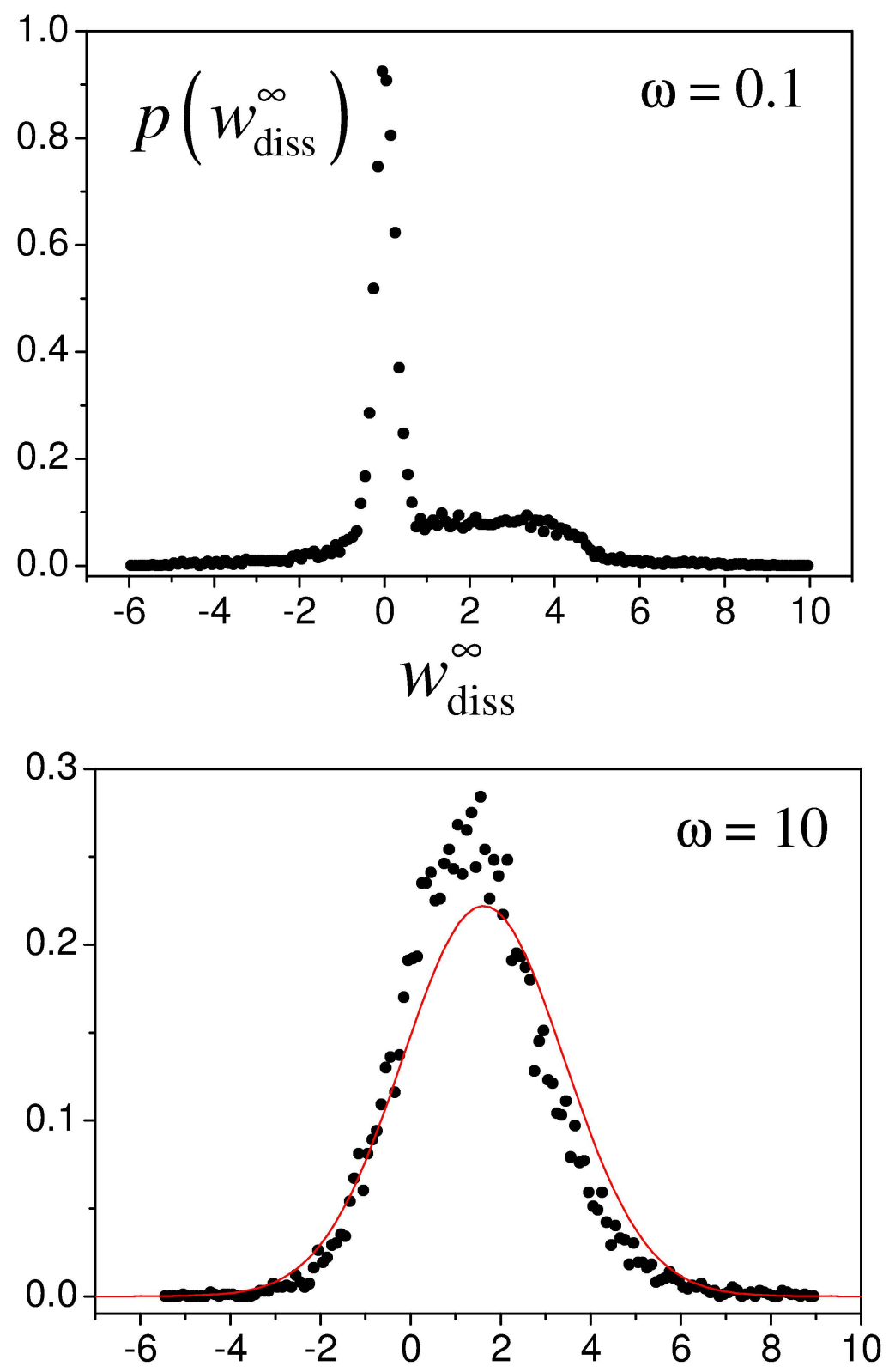
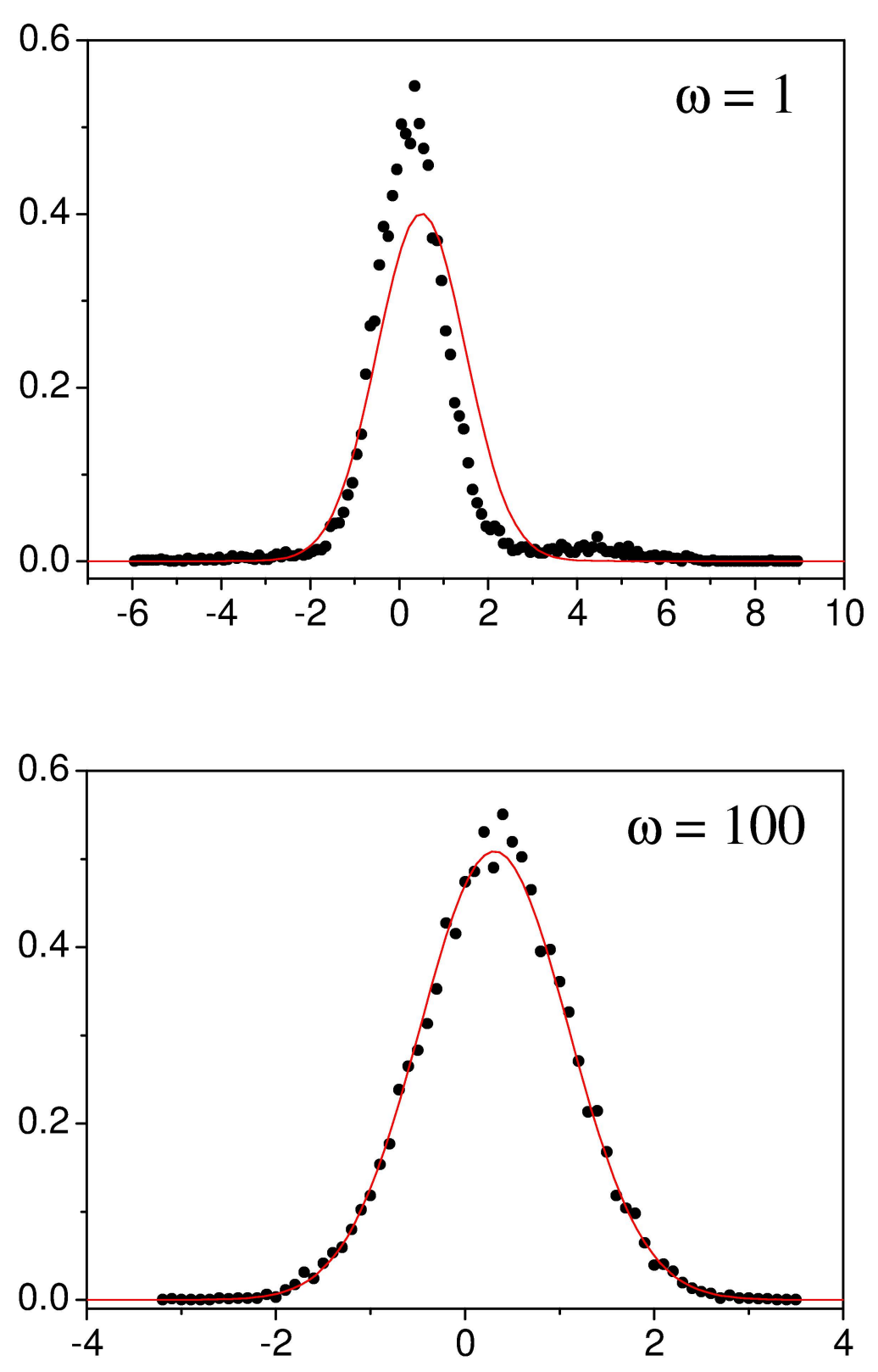
Case 1
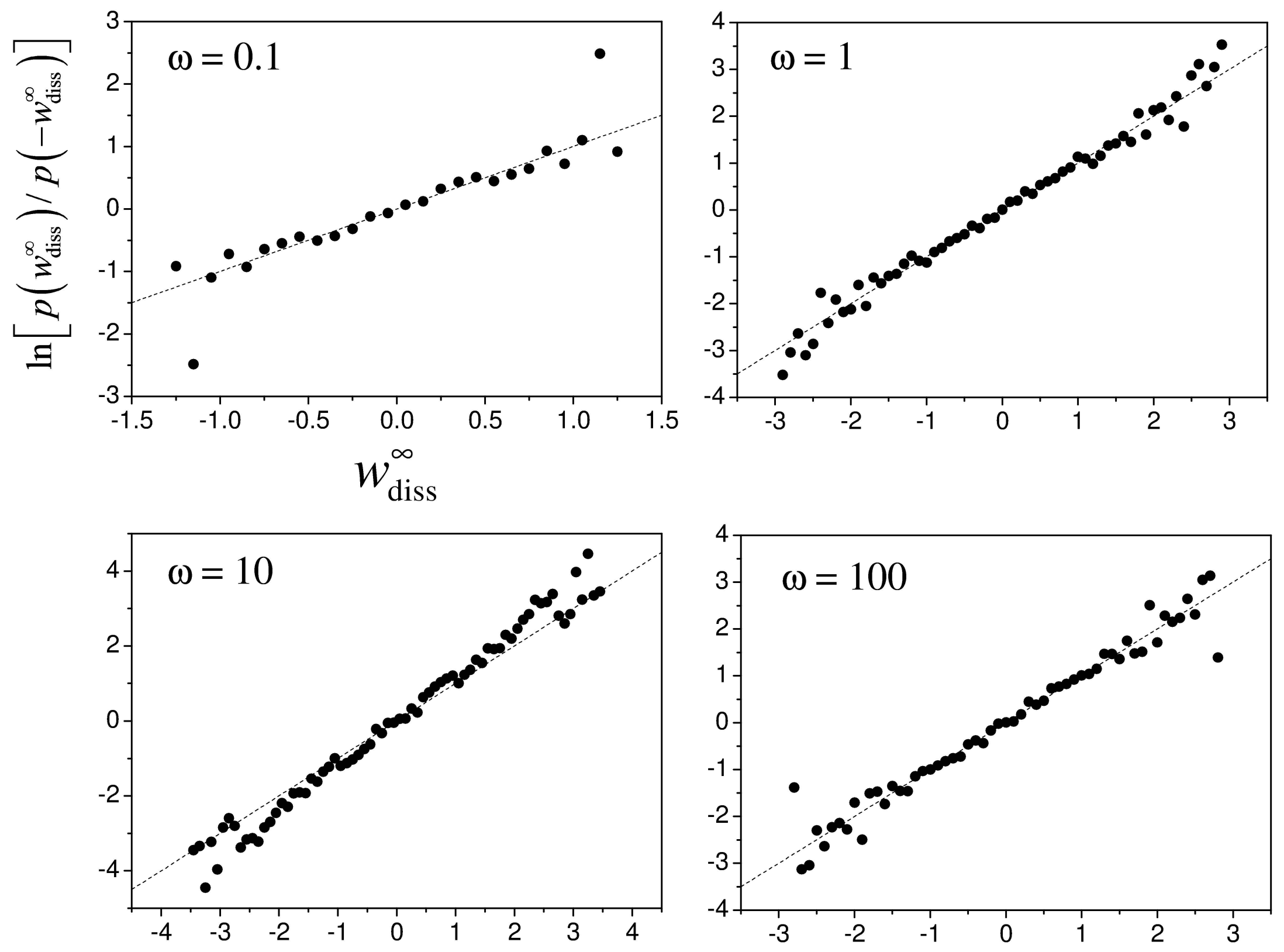
Case 2
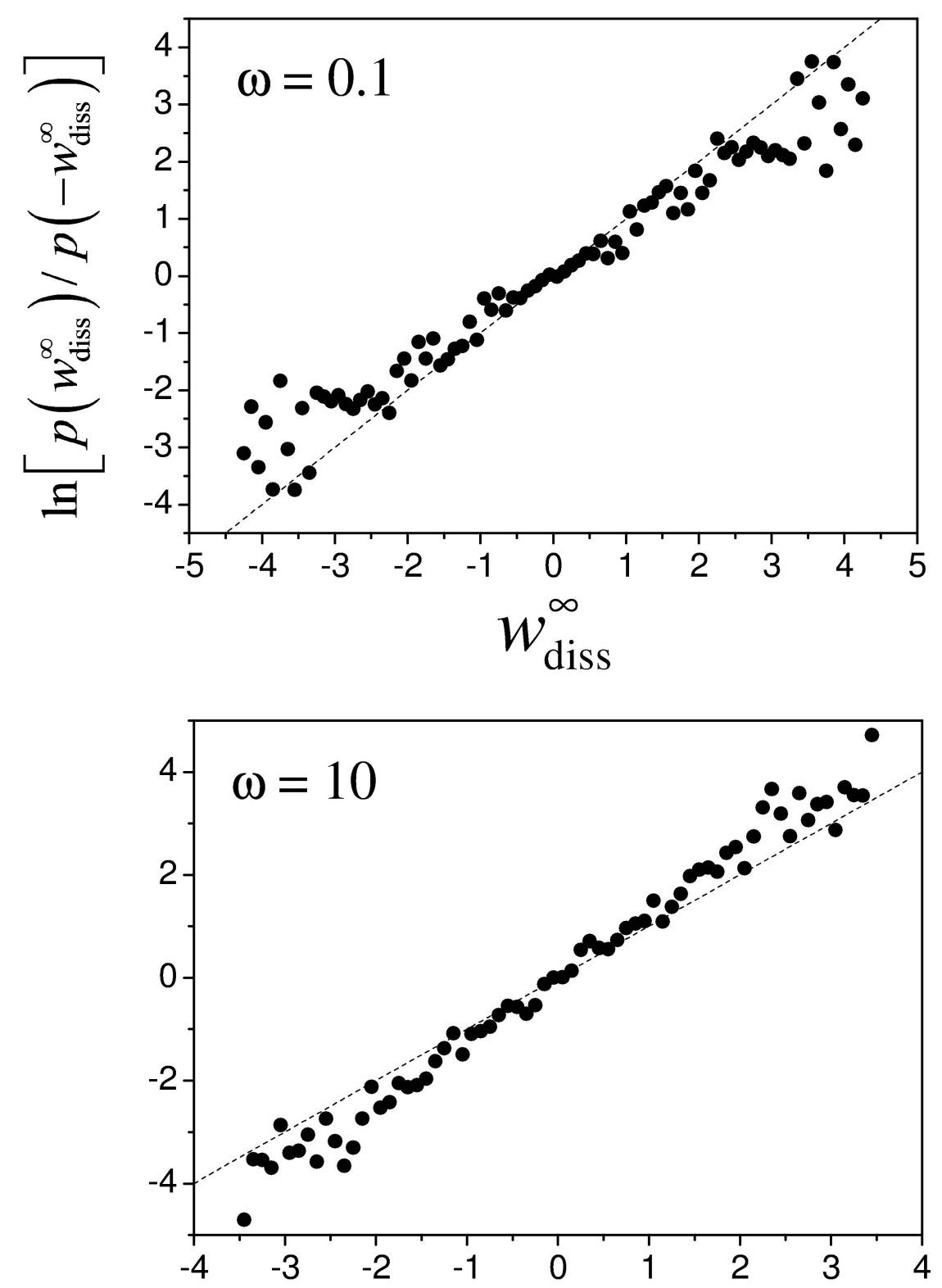
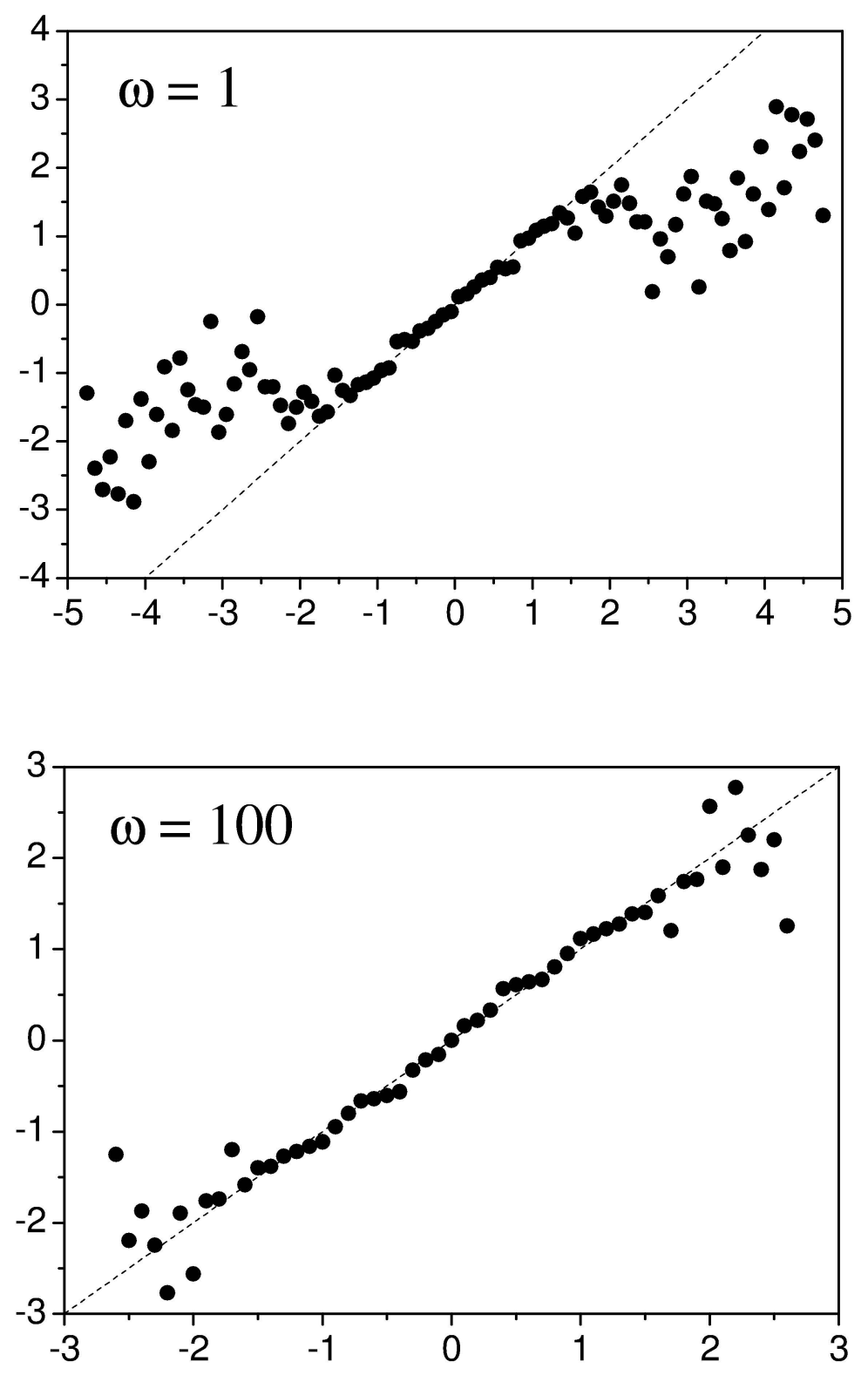